\title{
Manipulative and Manual Therapies in the Management of Patients with Prior Lumbar Surgery: A Systematic Review
}

Clinton J Daniels ( $\square$ clintdanielsdc@gmail.com )

VA Puget Sound Health Care System https://orcid.org/0000-0002-2727-6184

Zachary A. Cupler

VA Butler Healthcare

Jordan A Gliedt

Medical College of Wisconsin

Sheryl Walters

Logan University

Alec L Schielke

VA Palo Alto Health Care System

Nathan A Hinkeldey

VA Central lowa Health Care System

Derek J Golley

VA Western New York Healthcare System Buffalo VA Medical Center

Cheryl Hawk

Texas Chiropractic College

Research article

Keywords: Postsurgical, Failed back surgery syndrome, Postoperative periods, Spinal manipulation, Manual therapy

Posted Date: May 5th, 2020

DOI: https://doi.org/10.21203/rs.3.rs-26281/v1

License: @ (i) This work is licensed under a Creative Commons Attribution 4.0 International License. Read Full License

Version of Record: A version of this preprint was published at Complementary Therapies in Clinical Practice on February 1st, 2021. See the published version at https://doi.org/10.1016/j.ctcp.2020.101261. 


\section{Abstract}

Background

The purpose was to identify, summarize, and rate scholarly literature that describes manipulative and manual therapy following lumbar surgery.

\section{Methods}

The review was conducted in accordance with the Preferred Reporting Items for Systematic Reviews and Meta-Analyses (PRISMA) and was registered with PROSPERO. PubMed, Cochrane Database of Systematic Reviews, ICL, CINAHL, and PEDro were searched through July 2019. Articles were screened independently by at least two reviewers for inclusion. Articles included described the practice, utilization, and/or clinical decision making to post surgical intervention with manipulative and/or manual therapies. Data extraction consisted of principal findings, pain and function/disability, patient satisfaction, opioid/medication consumption, and adverse events. Scottish Intercollegiate Guidelines Network critical appraisal checklists were utilized to assess study quality.

\section{Results}

Literature search yielded 1916 articles, 348 duplicates were removed, 109 full-text articles were screened and 50 citations met inclusion criteria. There were 37 case reports/case series, 3 randomized controlled trials, 3 pilot studies, 5 systematic/scoping/narrative reviews, and 2 commentaries.

\section{Conclusion}

The findings of this review may help inform practitioners who utilize manipulative and/or manual therapies regarding levels of evidence for patients with prior lumbar surgery. Following lumbar surgery, the evidence indicated inpatient neural mobilization does not improve outcomes. There is inconclusive evidence to recommend for or against most manual therapies after most surgical interventions.

\section{Trial registration}

Prospectively registered with PROSPERO (\#CRD42020137314). Registered 24 January 2020.

\section{Background}

Low back pain is the leading cause of disability worldwide, impacting roughly 540 million people at any given time [1]. Lumbar surgical procedures have become increasingly more widespread over the past several decades. Surgical treatment for lumbar degenerative disc disease increased 2.4 -fold from 2000 to 2009 [2], and there were 1,288,496 new posterior lumbar fusion operations reported in the United States alone between 1998 and 2008 [3]. From $2004-2015$ the volume of elective lumbar fusion procedures in the United States rose by $62.3 \%$ (or 32.1 per 100,000$)$ from $122,679(60.4$ per 100,000$)$ to 199,140 (79.8 per $100,000)[4]$. The greatest increase in fusions occurred in adults over 65 years old, and octogenarians saw an increase from 1,144 fusions in 2004 to 2,061 fusions in $2013[4,5]$.

The most frequent condition considered appropriate for lumbar surgery is low back pain and radiculopathy secondary to lumbar disc herniation [6], with discectomy being the most commonly performed lumbar surgical procedure [7]. Recurrence of spinal or radicular symptoms is common following surgical intervention [8-10]. Following lumbar discectomy for symptomatic lumbar disc herniation the 1-year and 3-year recurrence rate for leg symptoms has been estimated to be $20 \%$ and $45 \%$ and for recurrent low back pain $29 \%$ and $65 \%$ respectively [11].

Postoperative pain and potential for future operative procedure is common for those undergoing lumbar surgical procedure. Patients that undergo lumbar discectomy procedure are 2.97 times more likely to require a future lumbar fusion than individuals without prior discectomy [12]. Failed back surgery syndrome (FBSS) is a regular indicator for spinal cord stimulator implant/neuromodulation [13], though may only provide pain relief for a portion of individuals undergoing this intervention. Turner et al. reported only $50-60 \%$ of failed back surgery patients with implanted neuromodulation reported $50 \%$ pain improvement and $40-50 \%$ continue to experience pain [13].

Many individuals with chronic pain complaints seek manual and manipulative therapy (MMT) for non-pharmacological pain management from chiropractors, osteopaths, physical therapists and massage therapists $[4,6,7,11,12]$. Manual therapy is the application of the practitioner's hands directly to soft tissues or joints using techniques such as mobilization, stretching, myofascial release, massage, and muscle energy techniques [14]. Manipulation is a type of manual therapy that involves the practitioner applying a high-velocity, low-amplitude manual force to a perceived hypo-mobile joint to approximate the joint near its end range of motion and to restore its physiological joint ROM [15], or alternatively through a table-assisted approach such as flexion-distraction (FD). MMT may be a potential treatment option to aid in pain reduction and functional preservation in those with a prior history of lumbar surgical intervention.

The authors are unaware of any prior systematic reviews analyzing the literature of MMT for individuals with a history of lumbar surgery. The primary aim of this study was to investigate the current relationship of MMT to the management of pain, function, patient satisfaction, and opioid/medication utilization for patients with prior lumbar operative procedures. A secondary purpose of this study was to assess the adverse events reported in the same body of literature.

\section{Methods}

\section{Search strategy}


A literature search was performed July 2019 of PubMed, Cochrane Database of Systematic Reviews, Index to Chiropractic Literature, Cumulative Index to Nursing and Allied Health Literature (CINAHL), Physiotherapy Evidence Database (PEDro) from inception of each database through July 2019. We combined numerous MMT search terms, with a variety of terms relevant to surgical interventions (Tables 1 and 2). The list of references of included publications was manually checked for additional studies potentially meeting the inclusion criteria.

Table 1

Search terms related to manipulation, manual therapy and surgical interventions

\begin{tabular}{|c|c|c|}
\hline Treatment Strategy & Prior Procedure & Condition/Region \\
\hline Chiropractic & Postsurgical & Failed Back Syndrome \\
\hline Musculoskeletal Manipulations & Postoperative & Spine \\
\hline Osteopathic Manipulations & Post-Surgical & Spinal \\
\hline Orthopedic Manipulations & Post-Operative & Low Back \\
\hline Manual Therapy & Fusion & Lumbar \\
\hline Manual Therapies & Decompression & Lumbosacral \\
\hline Manipulative Therapy & Lumbar Spine Surgery & Back Pain \\
\hline Manipulative Therapies & Microdiskectomy & Radiculopathy \\
\hline Manipulative Rehabilitation & Microdiscectomy & Radicular Pain \\
\hline Joint Manipulation & Discectomy & Sciatica \\
\hline Joint Mobilization & Diskectomy & Disc Herniation \\
\hline Mobilization Therapy & Laminectomy & Disk Herniation \\
\hline Spinal Mobilization & Laminotomy & Intervertebral Disc \\
\hline Soft Tissue Mobilization & Disc Replacement & Intervertebral Disk \\
\hline Flexion-Distraction & Disk Replacement & Disc Degeneration \\
\hline Myofascial & Vertebroplasty & Disk Degeneration \\
\hline Active Release & Kyphoplasty & Spinal Stenosis \\
\hline Graston & Foraminotomy & Spondylolisthesis \\
\hline Massage & Interlaminar Implant & Spondylosis \\
\hline Stretching Techniques & Spinal Cord Stimulator & Spondylolysis \\
\hline Muscle Stretching & Intrathecal drug delivery & Adjacent segment disease \\
\hline Static Stretching & Laser Surgery & Junction Failure \\
\hline Passive Stretching & Extreme Lateral Interbody & Degenerative Disc Disease \\
\hline Proprioceptive Neuromuscular Facilitation & & Degenerative Disk Disease \\
\hline PNF Stretching & & Scoliosis \\
\hline \multicolumn{3}{|l|}{ Post Isometric Relaxation } \\
\hline \multicolumn{3}{|l|}{ Contract-Relax } \\
\hline \multicolumn{3}{|l|}{ Instrument Assisted Soft Tissue } \\
\hline \multicolumn{3}{|l|}{ Instrument Assisted Manipulation } \\
\hline \multicolumn{3}{|l|}{ Instrument Assisted Adjustment } \\
\hline \multicolumn{3}{|l|}{ Instrument Assisted Adjusting } \\
\hline \multicolumn{3}{|l|}{ Manipulation Under Anesthesia } \\
\hline Spinal Manipulation & & \\
\hline
\end{tabular}


((((postsurgical OR postoperative OR post-surgical OR post-operative) AND (spine OR low back OR lumbar OR lumbosacral OR "back pain" OR radiculopathy OR radicular pain OR sciatica OR disc herniation OR disk herniation OR intervertebral disc OR intervertebral disk OR spinal OR degenerative disc disease OR degenerative disk disease OR disc degeneration OR disk degeneration OR scoliosis OR spinal stenosis OR spondylolisthesis OR spondylosis OR spondylolysis OR failed back syndrome OR adjacent segment disease OR joint failure)) OR (((fusion OR decompression) OR (laser AND (surgery or surgeries))) AND (spine OR low back OR lumbar OR lumbosacral OR "back pain" OR radiculopathy OR radicular pain OR sciatica OR disc herniation OR disk herniation OR intervertebral disc OR intervertebral disk OR spinal OR degenerative disc disease OR degenerative disk disease OR disc degeneration OR disk degeneration OR scoliosis OR spinal stenosis OR spondylolisthesis OR spondylosis OR spondylolysis OR failed back syndrome OR adjacent segment disease OR joint failure))) OR (failed back surgery syndrome OR lumbar spine surgery OR microdiscectomy OR microdiskectomy OR diskectomy OR discectomy OR laminectomy OR laminotomy OR disc replacement OR disk replacement OR vertebroplasty OR kyphoplasty OR

foraminotomy OR interlaminar implant OR "spinal cord stimulator" OR intrathecal drug delivery OR "extreme lateral interbody fusion")) AND (( (spinal manipulation OR chiropractic OR musculoskeletal manipulations OR osteopathic manipulation OR orthopedic manipulation OR manual therapy OR manual therapies) OR (manipulative AND (therapy OR therapies OR rehabilitation)) OR ("joint manipulation" OR "joint mobilization" OR "mobilization therapy" OR "spinal mobilization" OR "soft tissue mobilization" OR flexion distraction OR myofascial OR "active release" OR Graston OR massage OR stretching techniques OR muscle stretching OR static stretching OR passive stretching OR proprioceptive neuromuscular facilitation OR "PNF stretching" OR "post isometric relaxation" OR contract-relax) OR (instrument assisted AND (soft tissue OR manipulation OR adjusting OR adjustment)) OR "manipulation under anesthesia")) NOT ("Animals"[Mesh] NOT ("Animals"[Mesh] AND "Humans"[Mesh])) AND English[la]

Completed studies accepted for publication, but not yet in-print, were identified by searching clinicaltrials.gov and the World Health Organization International Clinical Trials Registry. The literature was searched with the assistance of a health sciences librarian (SW), and titles were screened independently by two different reviewers (CJD, ZAC). The review was conducted in accordance with the Preferred Reporting Items for Systematic Reviews and Meta-Analyses (PRISMA) and was registered with PROSPERO (\#CRD42020137314).

\section{Eligibility criteria}

The inclusion and exclusion criteria are available in Table 3. All research designs published by peer-reviewed scholarly journals in English were included in the search. Commentaries from non-peer reviewed sources (e.g., trade magazines) and other non-scholarly sources were excluded, as were writings not specific to post-surgical care provided. Case reports and case series were included to inform decision-making when no other higher level of evidence was available [16, 17]. Exclusions included animal studies as done by the method from the Cochrane Handbook for Systematic Reviews of Interventions [18]. Abstracts of conference proceedings were not included due to the high rate of conference presentations that never reach full publication [19]. Articles were considered for final inclusion if they describe the practice, utilization, and/or clinical decision making related to post-surgical intervention with MMT.

Table 3

Eligibility criteria

\begin{tabular}{|c|c|}
\hline Inclusion & Exclusion \\
\hline - Human subjects aged 18 or older & - Non-peer-reviewed publications \\
\hline - English language & - Conference abstracts \\
\hline \multirow{2}{*}{$\begin{array}{l}\text { - Intervention includes manipulation AND/OR manual therapy AND/OR } \\
\text { mobilization with or without multimodal approach }\end{array}$} & - Non-English language \\
\hline & - Animal studies \\
\hline $\begin{array}{l}\text { - Treatment of status post-surgical low back pain (degenerative or } \\
\text { traumatic) }\end{array}$ & - Study protocol \\
\hline \multirow{2}{*}{$\begin{array}{l}\text { - Surgery types (fusion, laminectomy, decompression, microdiscectomy, } \\
\text { disc replacement, veterbroplasty, spinal stimulators/implants, ...) }\end{array}$} & - Prior surgery for scoliosis \\
\hline & - Red flag condition identified which resulted in subsequent surgery \\
\hline \multirow{4}{*}{ - Any study design } & $\begin{array}{l}\text { - Surgical intervention performed as result of adverse event purportedly related } \\
\text { to manipulative and/or manual therapy }\end{array}$ \\
\hline & $\begin{array}{l}\text { - Unless patient already had a prior lumbar surgery predating } \\
\text { manipulative/manual therapy }\end{array}$ \\
\hline & - Dry needling/acupuncture \\
\hline & $\begin{array}{l}\text { - Non-surgical treatments which do not include manipulative OR manual } \\
\text { therapies (e.g. physical modalities, medications, braces and other equipment) }\end{array}$ \\
\hline
\end{tabular}

\section{P - Participants/population}

- Adults $\geq 18$ years old, prior lumbar surgical procedure for degenerative and/or traumatic condition.

I - Interventions

- Manipulation and/or manual therapy; may include multimodal care

C - Comparators

- No restrictions 
O- Outcomes

- Pain and function/disability

- Patient satisfaction

- Opioid and medication and consumption

- Note adverse events

\section{Methods of review Study selection}

The screening process was conducted independently by two authors, and coauthors were asked to contribute citations with which they were familiar but which might be missing from the formal search. Citations were screened by two reviewers by reading the title and abstract for each article. Abstracts of the citations that obviously or possibly met the review criteria were saved. The full papers of each abstract were retrieved and each article was reviewed independently by at least 2 authors to verify that it met the inclusion criteria. Disagreements on eligibility were resolved by discussion and adjudicated by a third author when necessary. Articles that did not meet the criteria were discarded and a note was made as to why they were excluded. Once an article was included, the citation, study design, principal findings, surgical intervention, manual/manipulative therapy, and adverse events were extracted.

\section{Data extraction}

Two authors completed data extraction for each of the included studies. One author served as the primary extractor and the second served as a secondary extractor confirming the findings. Any disagreements were resolved through discussions and if necessary, a third reviewer. Data were extracted into Microsoft Word tables grouped by type of study design. Items collected on the data extraction tables included: citation with first author and publication year, surgical history, MMT intervention, principle findings comparison, adverse events, and medication discussion. For randomized controlled trial (RCT) and cohort designs, we separated principle findings into comparison, outcome measures, results, and conclusions. For studies that involved multiple surgical types within an individual patient, we classified the surgical type from least-to-most aggressive or advanced approach in the order of discectomy, laminectomy, fusion, artificial disc replacement, and spinal cord stimulator, respectively. Studies that incorporated multiple surgical types without stratifying results by type were classified as undifferentiated.

\section{Evaluation of risk of bias}

Scottish Intercollegiate Guideline Network (SIGN) critical appraisal checklists [20] were utilized to assess for risk of bias (quality). All RCTs and systematic reviews (SR) included in this study were assessed with the corresponding checklist provided by SIGN, with at least 2 authors performing each quality assessment. Disagreements were resolved with discussion and a third reviewer was incorporated as appropriate. The SIGN checklist rates each article as "high quality, low risk of bias", "acceptable quality, moderate risk of bias", and "low quality, high risk of bias".

For the SR checklist, there are 12 items to score and quality is rated as: high, low risk of bias $>9$, acceptable, moderate risk of bias $6-9$, and low, high risk of bias $<6$ (Table 4). For the RCT checklist, there are 10 items to score and quality is rated as: high, low risk of bias $>8$, acceptable, moderate risk of bias $5-8$, and low, high risk of bias $<5$ (Table 5).

Table 4

Modified SIGN systematic review checklist [21]

\begin{tabular}{|ll|}
\hline 1.1 & The research question is clearly defined and the inclusion/exclusion criteria must be listed in the paper. (If "No" then reject) \\
\hline 1.2 & A comprehensive literature search is carried out (If "No" then reject) \\
\hline 1.3 & At least two people should have selected studies. \\
\hline 1.4 & At least two people should have extracted data. \\
\hline 1.5 & The status of publication was not used as an inclusion criterion. \\
\hline 1.6 & The excluded studies are listed. \\
\hline 1.7 & The relevant characteristics of the included studies are provided. \\
\hline 1.8 & The scientific quality of included studies was assessed and reported. \\
\hline 1.9 & Was the scientific quality of the included studies used appropriately? \\
\hline 1.11 & The likelihood of publication bias was assessed appropriately. \\
\hline 1.12 & Conflicts of interest are declared \\
\hline
\end{tabular}




\begin{tabular}{|ll|}
\hline 1.1 & The study addresses an appropriate and clearly focused question. \\
\hline 1.2 & The assignment of subjects to treatment groups is randomized \\
\hline 1.3 & An adequate concealment method is used. \\
\hline 1.4 & The design keeps subjects and investigators 'blind' about treatment allocation \\
\hline 1.6 & The treatment and control groups are similar at the start of the trial \\
\hline 1.7 & All relevant outcomes are measured in a standard, valid and reliable way \\
\hline 1.9 & $\begin{array}{l}\text { What percentage of the individuals or clusters recruited into each treatment arm of } \\
\text { the study dropped out before the study was completed? }\end{array}$ \\
\hline 1.10 & Where the study is carried out at more than one site, results are comparable for all sites. \\
\hline
\end{tabular}

\section{Strength of evidence}

The strength of evidence for recommendations was based upon the quality and quantity of evidence available and as has been demonstrated elsewhere [21] and modified from the UK evidence report [22, 23]. The criteria are outlined in Table 6 and describe high, moderate, and inconclusive strength of evidence, and favorable or unfavorable recommendation.

Table 6

Rating of evidence from randomized controlled trials and systematic reviews [21-23]

\begin{tabular}{|ll|}
\hline Quality and quantity of evidence & Rating \\
\hline Consistent results found in at least 2 low risk-of-bias studies & High \\
\hline Results of at least 1 low risk-of-bias study or at least 2 low risk-of-bias studies with some inconsistency of results & Moderate \\
\hline Only acceptable-quality studies with inconsistent results, or only high-risk of bias studies & Inconclusive \\
\hline
\end{tabular}

\section{Results}

A comprehensive database search identified 1913 citations, and 3 additional citations were added from the grey literature. After 348 duplicates were removed, 1568 citations were screened and 1459 were excluded by title and abstract as irrelevant. A review of the remaining 109 full-text articles resulted in 50 studies meeting inclusion criteria (Fig. 1)[24]. Fifty-nine articles were excluded with reasons provided in Table 7.

Table 7

Reasons for excluded articles from this review

\begin{tabular}{|ll|}
\hline Reason & Number of citations \\
\hline Not about MMT & $30[84-113]$ \\
Not lumbar postsurgical & $11[114-124]$ \\
Lacks relevant specifics & $7[125-131]$ \\
Related to scoliosis & $3[132-134]$ \\
Not peer-reviewed source & $2[135-136]$ \\
Conference abstract & $2[137-138]$ \\
Book chapter & $1[139]$ \\
Not English language & $1[140]$ \\
Unable to obtain & $1[141]$ \\
\hline
\end{tabular}

The majority of included studies were case reports or case series $(n=37)$, followed by RCTs $(n=6)$, SRs $(n=3)$, scoping review $(n=1)$, narrative review $(n=1)$, and commentaries $(n=2)$. The most common reason for exclusion was due to care not involving MMT $(n=30)$.

\section{Systematic reviews}

Three SRs met the inclusion criteria. One of the 3 was high quality [25], 1 was acceptable quality [26], and 1 was low quality [27] (Table 8). Two of the 3 included SRs described physical therapy and rehabilitation intervention in patients with undifferentiated lumbar surgery for degenerative conditions [25, 26], and 1 described care following lumbar fusion surgery [27]. Two of the 3 describe physical therapy (PT) and rehabilitation including, but not specific to, MMT $[26,27]$, and 1 specifically described neural mobilization techniques [25].

Page 6/30 
Table 8

Quality (risk-of-bias) assessment of included systematic reviews

\begin{tabular}{|c|c|c|c|c|c|c|c|c|c|c|c|c|c|c|}
\hline \multirow[t]{2}{*}{ First Author and Year Published } & \multicolumn{14}{|c|}{ Items on modified SIGN* Checklist } \\
\hline & 1 & 2 & 3 & 4 & 5 & 6 & 7 & 8 & 9 & 10 & 11 & 12 & Total & Quality** \\
\hline Basson 2017 & 1 & 1 & 1 & 1 & 1 & 1 & 1 & 1 & 1 & 1 & 1 & 1 & 12 & $\mathrm{H}$ \\
\hline Gilmore 2015 & 1 & 1 & 1 & 1 & 0 & 0 & 1 & 1 & 1 & 0 & 0 & 1 & 8 & A \\
\hline Madera 2017 & 1 & 1 & 1 & 1 & 0 & 0 & 0 & 0 & 0 & 0 & 0 & 1 & 5 & $\mathrm{~L}$ \\
\hline \multicolumn{15}{|c|}{ *SIGN, Scottish Intercollegiate Guideline Network } \\
\hline \multicolumn{15}{|c|}{${ }^{\star *}$ Quality: $\mathrm{H}=$ High, $\mathrm{A}=$ Acceptable, $\mathrm{L}=\mathrm{Low}$} \\
\hline
\end{tabular}

The high-quality and acceptable-quality reviews addressed rehabilitation after a variety of lumbar surgical types (e.g. discectomy, laminectomy, fusion). The high-quality SR investigated neural mobilization and included 69 studies, of which only 1 study that was postoperative low back pain [28], and concluded that inpatient neural mobilization in the 3 days following lumbar operation did not add benefit to usual care [25]. The acceptable-quality review analyzed inpatient PT including 4 studies, of which 1 was relevant to MMT [28] and it was the same study identified by the neural mobilization SR [26].

Following lumbar fusion, a low-quality review found insufficient evidence to make an argument for or against the inclusion of joint mobilization, nerve mobilization, or soft-tissue mobilization for lumbar fusion postoperative rehabilitation [27]. Despite insufficient evidence, among other treatments, the study authors recommended joint mobilization of the thoracic spine and hips to maintain posture and increase functional mobility, early neural mobilization to improve ROM by decreasing nerve tension, and soft-tissue mobilization to decrease post-surgical pain and swelling around the incision site.

\section{Randomized controlled trials}

Table 9 provides the RCTs risk of bias as high, acceptable, and low-quality studies and Table 10 presents the evidence. Of the 6 RCTs, 3 were pilots and were underpowered to make any conclusions regarding efficacy and were not rated for quality. Of the 3 remaining studies, 2 were rated high-quality [28, 29] and 1 was rated acceptable-quality [30].

Table 9

Risk-of-bias assessment of included randomized clinical trials

\begin{tabular}{|c|c|c|c|c|c|c|c|c|c|c|c|c|}
\hline \multirow[t]{2}{*}{ First Author and Year Published } & \multicolumn{12}{|c|}{ Items on SIGN* Checklist } \\
\hline & 1 & 2 & 3 & 4 & 5 & 6 & 7 & 8 & 9 & 10 & Total & Quality** \\
\hline Mannion 2007 & 1 & 1 & 1 & 0 & 1 & 1 & 1 & 1 & 1 & 1 & 9 & $\mathrm{H}$ \\
\hline Scrimshaw 2001 & 1 & 1 & 1 & 0 & 1 & 1 & 1 & 1 & 1 & 1 & 9 & $\mathrm{H}$ \\
\hline Timm 1994 & 1 & 0 & 1 & 0 & 0 & 1 & 1 & 1 & 1 & 0 & 6 & A \\
\hline Kim 2015 & \multicolumn{11}{|c|}{ Pilot Study - Not Powered } & NR \\
\hline Kim 2016 & \multicolumn{11}{|c|}{ Pilot Study - Not Powered } & NR \\
\hline Kim 2017 & \multicolumn{11}{|c|}{ Pilot Study - Not Powered } & NR \\
\hline \multicolumn{13}{|c|}{ *SIGN, Scottish Intercollegiate Guideline Network } \\
\hline \multicolumn{13}{|c|}{${ }^{\star \star}$ Quality: $\mathrm{H}=$ High, $\mathrm{A}=$ Acceptable, $\mathrm{L}=\mathrm{Low}, \mathrm{NR}=$ Not Rated } \\
\hline
\end{tabular}


Table 10

Randomized controlled trial evidence table

\begin{tabular}{|c|c|c|c|c|c|c|c|c|c|}
\hline $\begin{array}{l}\text { Citation } \\
\text { and } \\
\text { Quality }\end{array}$ & Participants & Surgical History & Intervention & Comparison & $\begin{array}{l}\text { Outcome } \\
\text { Measures }\end{array}$ & Results & Conclusion & $\begin{array}{l}\text { Adverse } \\
\text { Events }\end{array}$ & $\Lambda$ \\
\hline $\begin{array}{l}\text { Scrimshaw } \\
2001 \\
\text { High [28] }\end{array}$ & $\begin{array}{l}\mathrm{n}=81 ; \\
\text { mean age: CG } \\
=55 \\
\mathrm{TG}=59 \\
\text { Duration } \\
\mathrm{CG}(<6 \mathrm{wks})= \\
8 \\
\mathrm{CG}(6 \mathrm{wk}-6 \mathrm{mo}) \\
=14 \\
\mathrm{CG}(>6 \mathrm{mo})=2 \\
\mathrm{TG}(<6 \mathrm{wks})= \\
19 \\
\mathrm{TG}(6 \mathrm{wk}-6 \mathrm{mo}) \\
=14 \\
\mathrm{TG}(>6 \mathrm{mo})= \\
14\end{array}$ & $\begin{array}{l}\text { Discectomy, } \\
\text { Laminectomy, } \\
\text { Fusion }\end{array}$ & $\begin{array}{l}\text { TG: Inpatient } \\
\text { Neural } \\
\text { Mobilization } \\
\text { 2x/day for } 3 \\
\text { days with } \\
\text { different } \\
\text { protocol for } \\
\text { laminectomy } \\
\text { and } \\
\text { discectomy } \\
\text { versus fusion }\end{array}$ & $\begin{array}{l}\text { CG; Standard } \\
\text { postoperative } \\
\text { care }\end{array}$ & $\begin{array}{l}\text { GPE (7- } \\
\text { Point) } \\
\text { VAS (0- } \\
100 \mathrm{~mm}) \\
\text { McGill } \\
\text { QDS } \\
\text { RTW }\end{array}$ & $\begin{array}{l}\text { GPE: no } \\
\text { difference } \\
\text { between } \\
\text { groups } \\
\text { VAS: no } \\
\text { difference } \\
\text { between } \\
\text { groups } \\
\text { McGill: no } \\
\text { difference } \\
\text { between } \\
\text { groups } \\
\text { QDS: no } \\
\text { difference } \\
\text { between } \\
\text { groups } \\
\text { RTW: no } \\
\text { difference } \\
\text { between } \\
\text { groups }\end{array}$ & $\begin{array}{l}\text { Neural } \\
\text { mobilization did } \\
\text { not provide } \\
\text { additional } \\
\text { benefit to } \\
\text { standard care }\end{array}$ & $\begin{array}{l}\text { Not } \\
\text { Reported }\end{array}$ & $\Lambda$ \\
\hline $\begin{array}{l}\text { Mannion } \\
2007 \\
\text { High [29] }\end{array}$ & $\begin{array}{l}\mathrm{n}=151 \\
\begin{array}{l}\text { Mean age: } \mathrm{CG} \\
=66\end{array} \\
\text { TG1 = } 64 \\
\text { TG2 = } 65 \\
\text { Duration LBP: } \\
\text { CG }=132 \mathrm{mo} \\
\text { TG1 = 94mo } \\
\text { TG2 = } 126 \mathrm{mo} \\
\text { Duration LP } \\
\text { CG = } 33 \mathrm{mo} \\
\text { TG1 = } 34 \mathrm{mo} \\
\text { TG2 }=41 \mathrm{mo}\end{array}$ & $\begin{array}{l}\text { Laminotomy } \\
\text { Discectomy }\end{array}$ & $\begin{array}{l}\text { Both groups } 2 \\
\text { sessions/week } \\
\text { up to } 12 \text { weeks } \\
\text { TG1: Spine } \\
\text { Stabilization } \\
\text { Exercise } \\
\text { TG2: PT-Mixed } \\
\text { (among PT } \\
\text { techniques } \\
\text { included } \\
\text { Maitland, } \\
\text { Manual } \\
\text { Therapy, } \\
\text { Spinal } \\
\text { Mobilization, } \\
\text { Soft Tissue } \\
\text { Mobilization) }\end{array}$ & $\begin{array}{l}\text { CG: Self- } \\
\text { Management }\end{array}$ & $\begin{array}{l}\text { NPRS (0- } \\
10) \text { for } \\
\text { LBP and } \\
\text { LP } \\
\text { RMQ }\end{array}$ & $\begin{array}{l}\text { NPRS: } \\
\text { significant } \\
\text { reduction in } \\
\text { LBP and LP } \\
\text { following } \\
\text { surgery, no } \\
\text { between } \\
\text { group } \\
\text { differences; } \\
\text { slight } \\
\text { statistical } \\
\text { increase in LP } \\
\text { from } \\
\text { completion of } \\
\text { rehab phase } \\
\text { through 12mo } \\
\text { post-op } \\
\text { RMQ: all } \\
\text { scores } \\
\text { reduced } \\
\text { following } \\
\text { surgery, no } \\
\text { significant } \\
\text { differences } \\
\text { between } \\
\text { groups }\end{array}$ & $\begin{array}{l}\text { All groups } \\
\text { improved. No } \\
\text { significant } \\
\text { difference } \\
\text { between groups } \\
\text { in pain and self- } \\
\text { rated disability } \\
\text { at } 24 \text { months } \\
\text { after surgery }\end{array}$ & $\begin{array}{l}1 \text { TG1 } \\
\text { patient } \\
\text { dropped } \\
\text { out after } \\
2 \\
\text { sessions } \\
\text { due to } \\
\text { increased } \\
\text { pain }\end{array}$ & $\wedge$ \\
\hline
\end{tabular}

CG = Control Group, $\mathrm{CLBP}=$ chronic low back pain, $\mathrm{Ex}=$ exercise, $\mathrm{GPE}=$ global perceived effect, Grade III = large amplitude rhythmic oscillating mobilization, $\mathrm{G}$ small amplitude rhythmic oscillating, HEP = home exercise program, HVLA = high-velocity, low-amplitude manipulation, LBP = low back pain, LP = leg pain, Mc Pain Questionnaire, NPRS = Numerical Pain Rating Scale, ODI = Oswestry Disability Index, OMT = Osteopathic Manipulative Technique, PCS-SF = Physical Cor Score of 36-item Short-Form, QDS = Quebec Disability Scale, RMQ = Roland Morris Questionnaire, ROM = Range of Motion, RTW = Return to work, TG = Treatm VAS $=$ Visual Analog Scale for pain 


\begin{tabular}{|c|c|c|c|c|c|c|c|c|c|}
\hline $\begin{array}{l}\text { Citation } \\
\text { and } \\
\text { Quality }\end{array}$ & Participants & Surgical History & Intervention & Comparison & $\begin{array}{l}\text { Outcome } \\
\text { Measures }\end{array}$ & Results & Conclusion & $\begin{array}{l}\text { Adverse } \\
\text { Events }\end{array}$ & $\Lambda$ \\
\hline \multirow[t]{2}{*}{$\begin{array}{l}\text { Timm } \\
1994 \\
\text { Acceptable } \\
{[30]}\end{array}$} & $\begin{array}{l}\mathrm{n}=250 \\
\text { mean age CG } \\
=45 \mathrm{TG} 1=42 \\
\mathrm{TG} 2=42 \mathrm{TG} 3 \\
=44 \\
\mathrm{TG} 4=43 \\
\text { duration cLBP } \\
\text { before } \\
\text { surgery (years): } \\
\mathrm{CG}=1.8 \mathrm{TG} 1 \\
=2.1 \mathrm{TG}=1.8 \\
\mathrm{TG} 3=2.2 \mathrm{TG} 4 \\
=1.9 \text { surgery } \\
\text { to tx (years): } \\
\text { CG = 1.2 TG1 } \\
=1.3 \text { TG2 = } 1.5 \\
\text { TG3 = } 1.5 \text { TG4 } \\
=1.6\end{array}$ & L5 laminectomy & $\begin{array}{l}\text { All groups } \\
\text { 3x/week for } 8 \\
\text { weeks } \\
\text { TG1: physical } \\
\text { agents (hot } \\
\text { packs, } \\
\text { ultrasound, } \\
\text { TENS unit) } \\
\text { TG2: joint } \\
\text { manipulation } \\
\text { (large- } \\
\text { amplitude, } \\
\text { low-velocity } \\
\text { T12-S1 prone } \\
\text { (Grade Ill or } \\
\text { IV)) } \\
\text { TG3: low-tech } \\
\text { exercise } \\
\text { (McKenzie and } \\
\text { Spine } \\
\text { Stabilization) } \\
\end{array}$ & $\begin{array}{l}\text { CG: No } \\
\text { treatment }\end{array}$ & $\begin{array}{l}\text { modified- } \\
\text { modified } \\
\text { Schober } \\
\text { (lumbar } \\
\text { ROM) } \\
\text { Cybex } \\
\text { liftask } \\
\text { (strength) } \\
\text { ODI }\end{array}$ & $\begin{array}{l}\text { modified- } \\
\text { modified } \\
\text { Schober: low- } \\
\text { tech and high- } \\
\text { tech Ex } \\
\text { increased } \\
\text { lumbar flexion } \\
\text { and extension } \\
\text { ROM, Joint } \\
\text { manipulation } \\
\text { increased } \\
\text { extension } \\
\text { ROM } \\
\text { Cybex liftask: } \\
\text { low-tech and } \\
\text { high-tech Ex } \\
\text { increased } \\
\text { lifting force } \\
\text { output, no } \\
\text { difference } \\
\text { between } \\
\text { groups } \\
\text { ODI: low-tech } \\
\text { and high-tech } \\
\text { demonstrated } \\
\text { improved ODI, } \\
\text { no between } \\
\text { group } \\
\text { differences }\end{array}$ & & $\begin{array}{l}\text { None } \\
\text { reported. }\end{array}$ & $\Lambda$ \\
\hline & & & $\begin{array}{l}\text { TG4: high tech } \\
\text { exercise Large- } \\
\\
\\
\text { amplitude } \\
\text { (Bicycle } \\
\text { ergometry } \\
\text { followed by } \\
\text { Isotonic Ex on } \\
\text { Cybex TEF and } \\
\text { Torso) }\end{array}$ & & & & & & \\
\hline
\end{tabular}

$\mathrm{CG}=$ Control Group, $\mathrm{cLBP}=$ chronic low back pain, $\mathrm{Ex}=$ exercise, $\mathrm{GPE}=$ global perceived effect, Grade III = large amplitude rhythmic oscillating mobilization, $\mathrm{G}$ । small amplitude rhythmic oscillating, HEP = home exercise program, HVLA = high-velocity, low-amplitude manipulation, LBP = low back pain, LP = leg pain, Mc Pain Questionnaire, NPRS = Numerical Pain Rating Scale, ODI = Oswestry Disability Index, OMT = Osteopathic Manipulative Technique, PCS-SF = Physical Cor Score of 36-item Short-Form, QDS = Quebec Disability Scale, RMQ = Roland Morris Questionnaire, ROM = Range of Motion, RTW = Return to work, TG = Treatm VAS = Visual Analog Scale for pain 


\begin{tabular}{|c|c|c|c|c|c|c|c|c|c|}
\hline $\begin{array}{l}\text { Citation } \\
\text { and } \\
\text { Quality }\end{array}$ & Participants & Surgical History & Intervention & Comparison & $\begin{array}{l}\text { Outcome } \\
\text { Measures }\end{array}$ & Results & Conclusion & $\begin{array}{l}\text { Adverse } \\
\text { Events }\end{array}$ & \\
\hline $\begin{array}{l}\text { Kim } 2015 \\
\text { Not Rated } \\
{[72]}\end{array}$ & $\begin{array}{l}\mathrm{N}=33 ; \\
\text { Mean age: } \\
\text { TG1 = 46.4 } \\
\text { TG2 = 46.6 }\end{array}$ & $\begin{array}{l}\text { Lumbar } \\
\text { microdiscectomy }\end{array}$ & $\begin{array}{l}\text { TG1: OMT } \\
\text { including soft } \\
\text { tissue and } \\
\text { joint } \\
\text { mobilization, } \\
\text { myofascial } \\
\text { release, } \\
\text { neuromuscular } \\
\text { technique, } \\
\text { muscle energy } \\
\text { technique, } \\
\text { craniosacral } \\
\text { release and rib } \\
\text { raising and } \\
\text { mobilization } \\
\text { (not including } \\
\text { HVLA) }\end{array}$ & $\begin{array}{l}\text { TG2: Exercise } \\
\text { (1 week back } \\
\text { and } \\
\text { abdominal } \\
\text { stretching, } \\
\text { next } 2 \text { weeks } \\
\text { isometric } \\
\text { strengthening } \\
\text { back and } \\
\text { hips, final } \\
\text { week back } \\
\text { and stability } \\
\text { exercise } \\
\text { using Pilates } \\
\text { apparatus) }\end{array}$ & $\begin{array}{l}\text { RMQ } \\
\text { VAS Leg } \\
\text { Pain } \\
\text { VAS LBP } \\
\text { Lumbar } \\
\text { ROM } \\
\text { Use of } \\
\text { Medication }\end{array}$ & $\begin{array}{l}\text { RDQ: OMT } \\
\text { greater } \\
\text { reduction in } \\
\text { disability } \\
\text { VAS leg pain: } \\
\text { OMT greater } \\
\text { reduction } \\
\text { VAS LBP: } \\
\text { OMT greater } \\
\text { Improvements } \\
\text { both groups, } \\
\text { OMT greater } \\
\text { improvement } \\
\text { in extension } \\
\text { and L side } \\
\text { bending } \\
\text { Improvements } \\
\text { both groups, } \\
\text { with fewer } \\
\text { patients } \\
\text { needing } \\
\text { medication in } \\
\text { OMT group }\end{array}$ & $\begin{array}{l}\text { Pilot study } \\
\text { shows the } \\
\text { feasibility of a } \\
\text { future RCT to } \\
\text { investigate OMT } \\
\text { rehabilitation for } \\
\text { post-operative } \\
\text { management } \\
\text { after lumbar } \\
\text { microdiscectomy }\end{array}$ & $\begin{array}{l}\text { None } \\
\text { Reported }\end{array}$ & $\begin{array}{l}A \\
b \\
n \\
p \\
\text { s } \\
\text { a } \\
\text { ir } \\
\text { n } \\
\text { a } \\
\text { a } \\
\text { ri } \\
\text { s }\end{array}$ \\
\hline $\begin{array}{l}\text { Kim } 2016 \\
\text { Not Rated } \\
\text { [74] }\end{array}$ & $\begin{array}{l}\mathrm{N}=21 \\
\text { Mean age: } \\
\mathrm{CG}=54.9 \\
\mathrm{TG}=45.7\end{array}$ & $\begin{array}{l}\text { Open laser } \\
\text { microdiscectomy }\end{array}$ & $\begin{array}{l}\text { TG = OMT } \\
\text { including joint } \\
\text { mobilization, } \\
\text { soft tissue } \\
\text { release, } \\
\text { myofascial } \\
\text { release, } \\
\text { neuromuscular } \\
\text { technique, } \\
\text { muscle energy } \\
\text { technique (not } \\
\text { including } \\
\text { HVLA) }\end{array}$ & $\begin{array}{l}\text { CG }=\text { active } \\
\text { control } \\
\text { receiving } \\
\text { home } \\
\text { exercise } \\
\text { booklet and } \\
\text { verbal } \\
\text { instruction to } \\
\text { perform HEP } \\
2 x / \text { week for } 4 \\
\text { weeks }\end{array}$ & $\begin{array}{l}\text { RMQ } \\
\text { VAS LBP } \\
\text { VAS legs } \\
\text { PCS-SF }\end{array}$ & $\begin{array}{l}\text { RDQ: greater } \\
\text { improvement } \\
\text { with OMT } \\
\text { VAS LBP: } \\
\text { Both groups } \\
\text { improved, no } \\
\text { between } \\
\text { group } \\
\text { differences } \\
\text { VAS legs: } \\
\text { Greater } \\
\text { decrease with } \\
\text { OMT } \\
\text { PCS-SF: both } \\
\text { groups } \\
\text { slightly } \\
\text { improved, } \\
\text { greater with } \\
\text { OMT }\end{array}$ & $\begin{array}{l}\text { Pilot study } \\
\text { supports the } \\
\text { feasibility of a } \\
\text { future RCT and } \\
\text { indicates OMT } \\
\text { rehabilitation } \\
\text { may be } \\
\text { important part of } \\
\text { post-operative } \\
\text { care after open } \\
\text { laser } \\
\text { discectomy. }\end{array}$ & $\begin{array}{l}\text { None } \\
\text { Reported }\end{array}$ & $\begin{array}{l}A \\
b \\
n \\
p \\
p \\
a \\
\text { ir } \\
n \\
a \\
a \\
\text { d } \\
\text { tl }\end{array}$ \\
\hline $\begin{array}{l}\text { Kim } 2017 \\
\text { Not Rated } \\
{[73]}\end{array}$ & $\begin{array}{l}\mathrm{N}=21 \\
\text { Mean age: } \\
\text { CG: } 54.9 \\
\text { TG: } 45.7\end{array}$ & $\begin{array}{l}\text { Lumbar } \\
\text { microdiscectomy }\end{array}$ & $\begin{array}{l}\text { TG: OMT } \\
\text { including soft } \\
\text { tissue and } \\
\text { joint } \\
\text { mobilization, } \\
\text { counter-strain } \\
\text { techniques, } \\
\text { neuromuscular } \\
\text { technique, } \\
\text { muscle energy } \\
\text { technique (not } \\
\text { including } \\
\text { HVLA) }\end{array}$ & $\begin{array}{l}C G=\text { active } \\
\text { control } \\
\text { receiving } \\
\text { home } \\
\text { exercise } \\
\text { booklet }\end{array}$ & $\begin{array}{l}\text { RMQ } \\
\text { VAS LBP } \\
\text { VAS legs }\end{array}$ & $\begin{array}{l}\text { RMQ: OMT } \\
\text { group } \\
\text { improved and } \\
\text { CG worsened } \\
\text { VAS LBP: } \\
\text { OMT group } \\
\text { improved and } \\
\text { CG worsened } \\
\text { VAS legs: } \\
\text { OMT group } \\
\text { improved and } \\
\text { CG worsened }\end{array}$ & $\begin{array}{l}\text { Demonstrated } \\
\text { potential of } \\
\text { manipulative } \\
\text { rehabilitation to } \\
\text { post-operative } \\
\text { management } \\
\text { after lumbar disc } \\
\text { surgery. } \\
\text { Definitive trials } \\
\text { with large same } \\
\text { sizes needed to } \\
\text { confirm } \\
\text { feasibility and } \\
\text { potential } \\
\text { therapeutic } \\
\text { effect. }\end{array}$ & $\begin{array}{l}\text { None } \\
\text { reported }\end{array}$ & $\begin{array}{l}\mathrm{E} \\
\mathrm{u} \\
\mathrm{p} \\
\mathrm{s} \\
\mathrm{a} \\
\text { ir } \\
\mathrm{n} \\
\mathrm{a} \\
\mathrm{a} \\
\mathrm{r}\end{array}$ \\
\hline
\end{tabular}

$\mathrm{CG}=$ Control Group, $\mathrm{cLBP}=$ chronic low back pain, $\mathrm{Ex}=$ exercise, $\mathrm{GPE}=$ global perceived effect, Grade III = large amplitude rhythmic oscillating mobilization, GI small amplitude rhythmic oscillating, HEP = home exercise program, HVLA = high-velocity, low-amplitude manipulation, LBP = low back pain, LP = leg pain, Mc Pain Questionnaire, NPRS = Numerical Pain Rating Scale, ODI = Oswestry Disability Index, OMT = Osteopathic Manipulative Technique, PCS-SF = Physical Cor Score of 36-item Short-Form, QDS = Quebec Disability Scale, RMQ = Roland Morris Questionnaire, ROM = Range of Motion, RTW = Return to work, TG = Treatm VAS = Visual Analog Scale for pain

Following lumbar surgery (undifferentiated), one RCT [29] compared a control group of self-management to 2 PT groups, a "spinal stabilization exercise group" and a "mixed-physical therapy group" including Maitland, manual therapy, spinal mobilization, and soft-tissue mobilization among other PT techniques. They found no between-group differences as measured by the numerical pain rating scale or Roland-Morris Disability Questionnaire. The study did not control for specific interventions utilized by physical therapists in treatment. This RCT was rated high quality (low-risk-of bias) by the SIGN checklist.

A second high-quality RCT was described above and investigated inpatient use of neural mobilization following undifferentiated lumbar surgery [28]. Their study found no between-group differences for global perceived effect, the visual analog scale for pain, McGill Pain Questionnaire, Quebec Disability Scale, or 
return-to-work.

The last RCT studied outcomes after L5 laminectomy in a 5-arm trial comparing control (no treatment) to postoperative physical agents, joint mobilization, low-tech exercise, and high-tech exercise [30]. This study was graded acceptable-quality as it did not adequately address group assignment randomization, blinding of the investigators or patients, and handling of missing data (intention-to-treat). In their study, active approaches were the most effective for the improvement of functional measures of chronic low back pain, with low-tech exercise having the longest interval of chronic low back pain relief. Joint mobilization increased lumbar extension ROM but did not impact objective outcomes for spinal function.

\section{Literature reviews, case reports, and commentaries}

There was a large body of lower-level studies that were not assessed for quality. This included 1 scoping review [31], 1 narrative review [32], 14 case series [33-46], 23 case reports [47-69], and 2 commentaries [70,71]. Ten of the case reports described 53 cases following discectomy, 16 reports described MMT in 143 cases post-laminectomy, 16 reports described MMT care for 67 cases after fusion, 1 report discussed post-surgical treatment in 8 cases after artificial disc replacement, and 1 report discussed care in 3 cases following implantation of spinal cord stimulators. There were multiple instances where a single report described FBSS cases from following more than 1 surgical intervention. The narrative review discusses lumbar fusion with relevance to chiropractors and the scoping review analyzed rehabilitation protocols directed at the lumbar spine in the perioperative periods. The findings from these studies and case reports are presented in Tables 11-13.

Table 11

Summary of included narrative, scoping and systematic reviews

\begin{tabular}{|c|c|c|c|}
\hline $\begin{array}{l}\text { First Author, Year } \\
\text { Published }\end{array}$ & Design & Quality & Principal Findings \\
\hline \multirow[t]{3}{*}{ Basson 2017[25] } & \multirow[t]{3}{*}{ SR } & \multirow[t]{3}{*}{ High } & - Systematic review and meta-analysis of neural mobilization for neuromusculoskeletal conditions \\
\hline & & & - 21 included in qualitative analysis, 1 related to post-lumbar surgery \\
\hline & & & - Neural mobilization did not provide added benefit to usual medical care \\
\hline \multirow[t]{3}{*}{ Daniels 2016[32] } & \multirow[t]{3}{*}{ NR } & \multirow[t]{3}{*}{ Not Rated } & - Describes indications for fusion, common surgical practice, and potential fusion complications \\
\hline & & & $\begin{array}{l}\text { - Patients with LBP and prior lumbar fusion may benefit with chiropractic manipulation, flexion-distraction, } \\
\text { or manipulation under anesthesia. }\end{array}$ \\
\hline & & & $\begin{array}{l}\text { - Large-scale RCTs are needed to effectively assess the safety and efficacy of chiropractic care for } \\
\text { patients after lumbar fusion }\end{array}$ \\
\hline \multirow[t]{5}{*}{ Gilmore 2015[26] } & \multirow[t]{5}{*}{ SR } & \multirow[t]{5}{*}{ High } & - Systematic review of physical therapy before and after surgery for lumbar degenerative condition \\
\hline & & & - 4 studies met inclusion criteria \\
\hline & & & - No clear benefit or risk of harm from performing either prone or side-lying transfers \\
\hline & & & $\begin{array}{l}\text { - Very-low-quality evidence suggests that physiotherapy may improve pain and function following lumbar } \\
\text { surgery }\end{array}$ \\
\hline & & & $\begin{array}{l}\text { - Further research into patient mobility, exercise and provision of education is required using outcome } \\
\text { measures that allow for comparison of results }\end{array}$ \\
\hline \multirow[t]{4}{*}{ Madera 2017[27] } & \multirow[t]{4}{*}{ SR } & \multirow[t]{4}{*}{ Acceptable } & - Review of existing literature regarding rehabilitation following lumbar fusion surgery \\
\hline & & & - 21 articles met the author's inclusion criteria \\
\hline & & & - Few articles offered specific rehabilitation protocols \\
\hline & & & $\begin{array}{l}\text { - Based on their review, the authors recommended immediate mobilization, followed by formal active } \\
\text { rehabilitation } 2-3 \text { months post-operatively }\end{array}$ \\
\hline \multirow[t]{5}{*}{ Marchand 2016[31] } & \multirow[t]{5}{*}{ ScR } & \multirow[t]{5}{*}{ Not Rated } & $\begin{array}{l}-28 \text { articles: comparing rehabilitation with placebo, no treatment, or another active treatment, or } \\
\text { rehabilitation combined with interventions. }\end{array}$ \\
\hline & & & - Outcomes: VAS, mODI, RMD, SLR. strength and endurance testing. \\
\hline & & & $\begin{array}{l}\text { - Following discectomy, early passive and active hip and knee flexion exercises were found to reduce time } \\
\text { to independent mobility and return to work }\end{array}$ \\
\hline & & & - No mention of MMT for fusion, or vertebral decompression \\
\hline & & & $\begin{array}{l}\text { - No conclusion could be drawn but notably multimodal programs including combinations of exercise, } \\
\text { education, group exchange, and ergonomics }\end{array}$ \\
\hline
\end{tabular}


Table 12

Evidence table for included case series or reports of patients with prior lumbar surgery

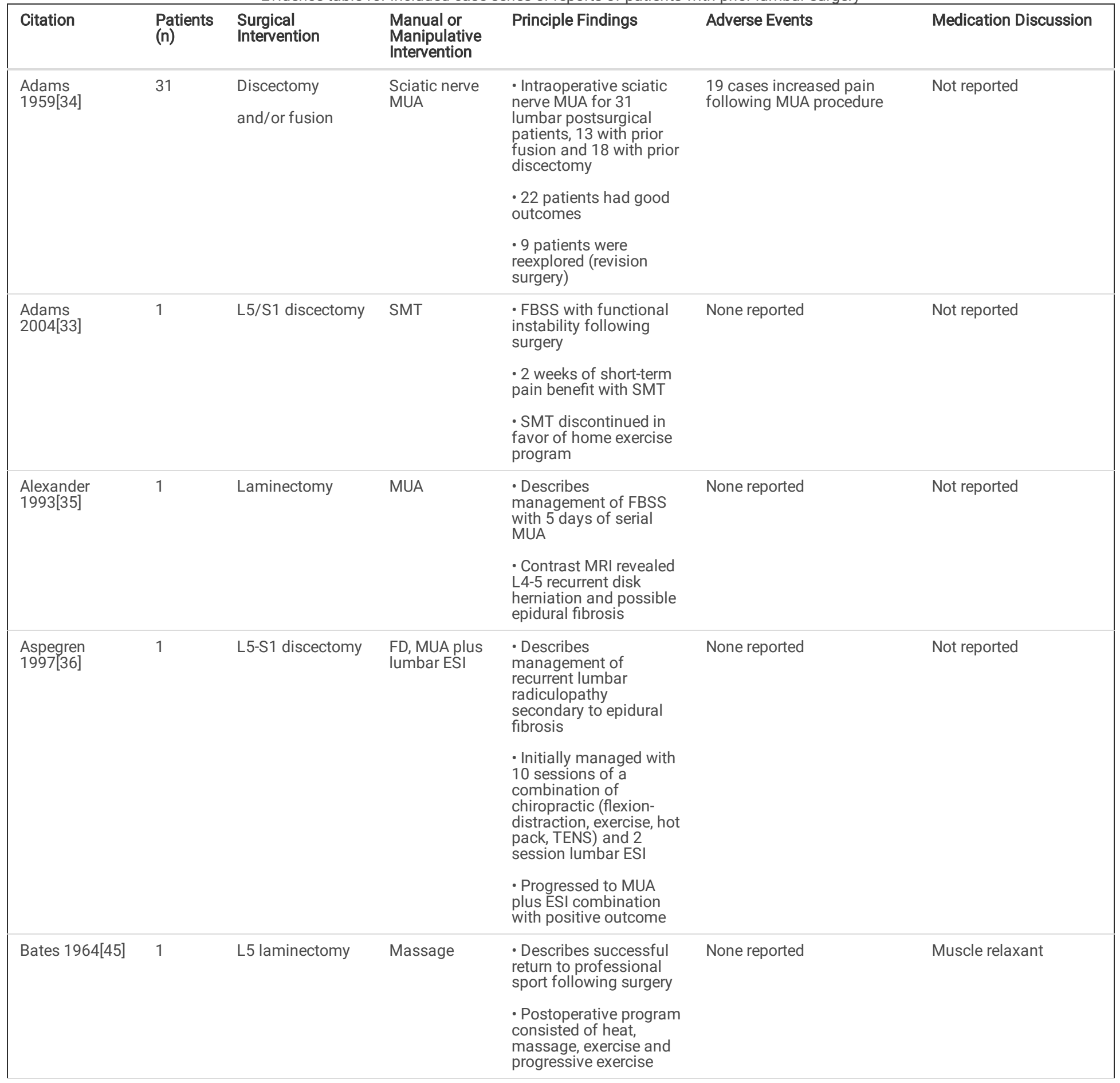

Abbreviations: ADL, activity of daily living; bid, bis in die (two times per day); cLBP, chronic low back pain; DTR, deep tendon reflexes; ESI, epidural steroid injection; FABQ, Fear-Avoidance Belief Questionnaire; FBSS, failed back surgery syndrome; FD, flexion distraction technique; FRI, Functional Rating Index; LBP, low back pain; LE, lower extremity; MUA, manipulation under anesthesia; NPS, numerical pain scale; NRS, numerical rating scale; NSAID, non-steroidal anti-inflammatory drug; ODI, Oswestry Disability Index; OMT, osteopathic manipulative therapy; OTC, over-the-counter; ROM, range of motion; RDQ, RolandMorris Disability Questionnaire; SCS, spinal cord stimulator; SMT, spinal manipulative therapy (Grade V Maitland); TX, treatment 


\begin{tabular}{|c|c|c|c|c|c|c|}
\hline Citation & $\begin{array}{l}\text { Patients } \\
\text { (n) }\end{array}$ & $\begin{array}{l}\text { Surgical } \\
\text { Intervention }\end{array}$ & $\begin{array}{l}\text { Manual or } \\
\text { Manipulative } \\
\text { Intervention }\end{array}$ & Principle Findings & Adverse Events & Medication Discussion \\
\hline $\begin{array}{l}\text { Benningfield } \\
\text { 1997[37] }\end{array}$ & 1 & $\begin{array}{l}\text { L5/S1 discectomy } \\
\text { with laminotomy }\end{array}$ & SMT & $\begin{array}{l}\text { - Describes } \\
\text { management of } \\
\text { recurrent LE radiation of } \\
\text { pain } 1 \text { year } \\
\text { postoperative } \\
\text { - TX consisted of SMT } \\
\text { and lumbar MedX } \\
\text { lumbar-extension } \\
\text { machine } \\
\text { - } 2 x \text { week } 6 \text { weeks, } \\
\text { followed by } 1 \times \text { week } 6 \\
\text { weeks } \\
\\
\cdot 30 \% \text { improvement in } \\
\text { strength }\end{array}$ & Not reported & $\begin{array}{l}\text { Aspirin, Tylenol 3, } \\
\text { lbuprofen with minimal } \\
\text { relief; } \\
\text { No post-TX reporting }\end{array}$ \\
\hline $\begin{array}{l}\text { Cornelson } \\
2018[61]\end{array}$ & 1 & $\begin{array}{l}\text { Multiple: fusion } \\
\text { and laminectomy } \\
\text { at L3-4 and L4-5 }\end{array}$ & $\begin{array}{l}\text { Neural } \\
\text { mobilization }\end{array}$ & $\begin{array}{l}\text { - Describes successful } \\
\text { management of patient } \\
\text { with adhesive } \\
\text { arachnoiditis following } \\
3 \text { lumbar procedures } \\
\text { - } 3 \text { weeks of neural } \\
\text { mobilization } \\
\text { - VAS reduced by } 2 \\
\text { points, straight leg raise } \\
\text { pain free, ODI reduced } \\
\text { from } 63-44 \% \text {, and } \\
\text { increased tolerance for } \\
\text { exercise }\end{array}$ & None reported & $\begin{array}{l}\text { Pre-TX Ibuprofen } 400- \\
600 \mathrm{mg} \text { per day; No } \\
\text { post-TX reporting }\end{array}$ \\
\hline Coulis 2013[51] & 2 & $\begin{array}{l}\text { Case } 1 \text { L5 } \\
\text { laminectomy with } \\
\text { left L4-5 } \\
\text { decompression } \\
\text { and right L5-S1 } \\
\text { decompression; } \\
\text { Case 2 } \\
\text { discectomy }\end{array}$ & $\begin{array}{l}\text { Case } 1 \text { FD, } \\
\text { case } 2 \text { FD } \\
\text { and SMT }\end{array}$ & $\begin{array}{l}\text { - Describes positive } \\
\text { benefits of SMT and FD } \\
\text { for patients with } \\
\text { laminectomy and } \\
\text { discectomy } \\
\text { - Case } 1 \text { reduced VAS } \\
6 / 10 \text { to } 4 / 10 \text { and } \\
\text { improvements in } \\
\text { function and ADLs } \\
\text { without exacerbation } \\
\text { - Case } 2 \text { no change in } \\
\text { VAS, but functional } \\
\text { improvement including } \\
\text { walking and driving } \\
\text { tolerance }\end{array}$ & $\begin{array}{l}\text { Case } 1 \text { none reported; Case } \\
2 \text { mild lumbar spine } \\
\text { soreness following initial TX } \\
\text { with non subsequent } \\
\text { adverse event }\end{array}$ & $\begin{array}{l}\text { Case } 1 \text { Diclofenac and } \\
\text { Cyclobenzaprine; Case } \\
2 \text { tricyclic } \\
\text { antidepressants, } \\
\text { acetaminophen, } \\
\text { meloxicam, } \\
\text { cyclobenzaprine and } \\
\text { opioids; No post-TX } \\
\text { reporting }\end{array}$ \\
\hline Cox 2009[55] & 1 & L4-S1 Fusion & FD & $\begin{array}{l}\text { - } 20 \text { sessions of FD } \\
\text { provided improvement } \\
\text { in pain and function } \\
\text { (ODI) } \\
\text { - LE pain completely } \\
\text { relieved and mild LBP } \\
\text { with use remained }\end{array}$ & None reported & Not reported \\
\hline
\end{tabular}

Abbreviations: ADL, activity of daily living; bid, bis in die (two times per day); cLBP, chronic low back pain; DTR, deep tendon reflexes; ESI, epidural steroid injection; FABQ, Fear-Avoidance Belief Questionnaire; FBSS, failed back surgery syndrome; FD, flexion distraction technique; FRI, Functional Rating Index; LBP, low back pain; LE, lower extremity; MUA, manipulation under anesthesia; NPS, numerical pain scale; NRS, numerical rating scale; NSAID, non-steroidal anti-inflammatory drug; ODI, Oswestry Disability Index; OMT, osteopathic manipulative therapy; OTC, over-the-counter; ROM, range of motion; RDQ, RolandMorris Disability Questionnaire; SCS, spinal cord stimulator; SMT, spinal manipulative therapy (Grade V Maitland); TX, treatment 


\begin{tabular}{|c|c|c|c|c|c|c|}
\hline Citation & $\begin{array}{l}\text { Patients } \\
\text { (n) }\end{array}$ & $\begin{array}{l}\text { Surgical } \\
\text { Intervention }\end{array}$ & $\begin{array}{l}\text { Manual or } \\
\text { Manipulative } \\
\text { Intervention }\end{array}$ & Principle Findings & Adverse Events & Medication Discussion \\
\hline $\begin{array}{l}\text { Demetrious } \\
2007[54]\end{array}$ & 1 & $\begin{array}{l}\text { Fusion, } 6 \text { lumbar } \\
\text { procedures }\end{array}$ & $\begin{array}{l}\text { FD, manual } \\
\text { trigger point } \\
\text { therapy }\end{array}$ & $\begin{array}{l}\text { - Pre-TX severe } \\
\text { compromise of ADLs } \\
\text { and total disability } \\
\text { status } \\
\text { - Improvement reported } \\
\text { for ADLs (ODI) and pain } \\
\text { (VAS) } \\
\text { - Workers compensation } \\
\text { ended trial of } \\
\\
\text { chiropractic care } \\
\text { despite apparent benefit }\end{array}$ & None reported & Not reported \\
\hline $\begin{array}{l}\text { Francio } \\
2017[65]\end{array}$ & 1 & Laminectomy & SMT & $\begin{array}{l}\text { - Describes successful } \\
\text { management post- } \\
\text { laminectomy with } \\
\text { combination of SMT } \\
\text { and McKenzie method } \\
\text { exercise } \\
\text { - Stable functional } \\
\text { improvement with no } \\
\text { significant pain or } \\
\text { disability (ODI) at 3- } \\
\text { month follow-up }\end{array}$ & None reported & $\begin{array}{l}\text { Non-responsive to OTC } \\
\text { medications, muscle } \\
\text { relaxants and pain } \\
\text { medicine }\end{array}$ \\
\hline Gluck 1996[56] & 1 & Discectomy & $\begin{array}{l}\text { FD, manual } \\
\text { therapy, SMT }\end{array}$ & $\begin{array}{l}\text { - Describes multimodal } \\
\text { treatment approach } \\
\text { emphasizing active } \\
\text { rehabilitation } \\
\text { techniques } \\
\text { - Transitioned from } \\
\text { passive therapy after } \\
\text { active patient was } \\
\text { deemed "permanent } \\
\text { and stationary" } \\
\text { - Improved lumbar ROM, } \\
\text { reduced pain (VAS) } 6.5 \\
\text { to } 3.8 \text {, reduced disability } \\
\text { (ODI) } 82-58 \% \text {, } \\
\text { improved ambulation } \\
\text { no longer required } \\
\text { assistive device, } \\
\text { improved sleep quality }\end{array}$ & None reported & $\begin{array}{l}\text { Meperidine (Demerol), } \\
\text { Motrin; Patient stopped } \\
\text { using pain medication } \\
\text { during treatment plan }\end{array}$ \\
\hline $\begin{array}{l}\text { Greenwood } \\
2012[58]\end{array}$ & 1 & $\begin{array}{l}\text { Fusion, } \\
\text { vertebrectomy, } \\
\text { cage } \\
\text { reconstruction }\end{array}$ & FD & $\begin{array}{l}\text { - Describes successful } \\
\text { management of chronic } \\
\text { low back pain } \\
\text { associated with } \\
\text { adjacent segment } \\
\text { disease } \\
\text { - Aviation crash survivor } \\
\text { with multilevel lumbar } \\
\text { fusion }\end{array}$ & None reported & Not reported \\
\hline
\end{tabular}

Abbreviations: ADL, activity of daily living; bid, bis in die (two times per day); cLBP, chronic low back pain; DTR, deep tendon reflexes; ESI, epidural steroid injection; FABQ, Fear-Avoidance Belief Questionnaire; FBSS, failed back surgery syndrome; FD, flexion distraction technique; FRI, Functional Rating Index; LBP, low back pain; LE, lower extremity; MUA, manipulation under anesthesia; NPS, numerical pain scale; NRS, numerical rating scale; NSAID, non-steroidal anti-inflammatory drug; ODI, Oswestry Disability Index; OMT, osteopathic manipulative therapy; OTC, over-the-counter; ROM, range of motion; RDQ, RolandMorris Disability Questionnaire; SCS, spinal cord stimulator; SMT, spinal manipulative therapy (Grade V Maitland); TX, treatment 


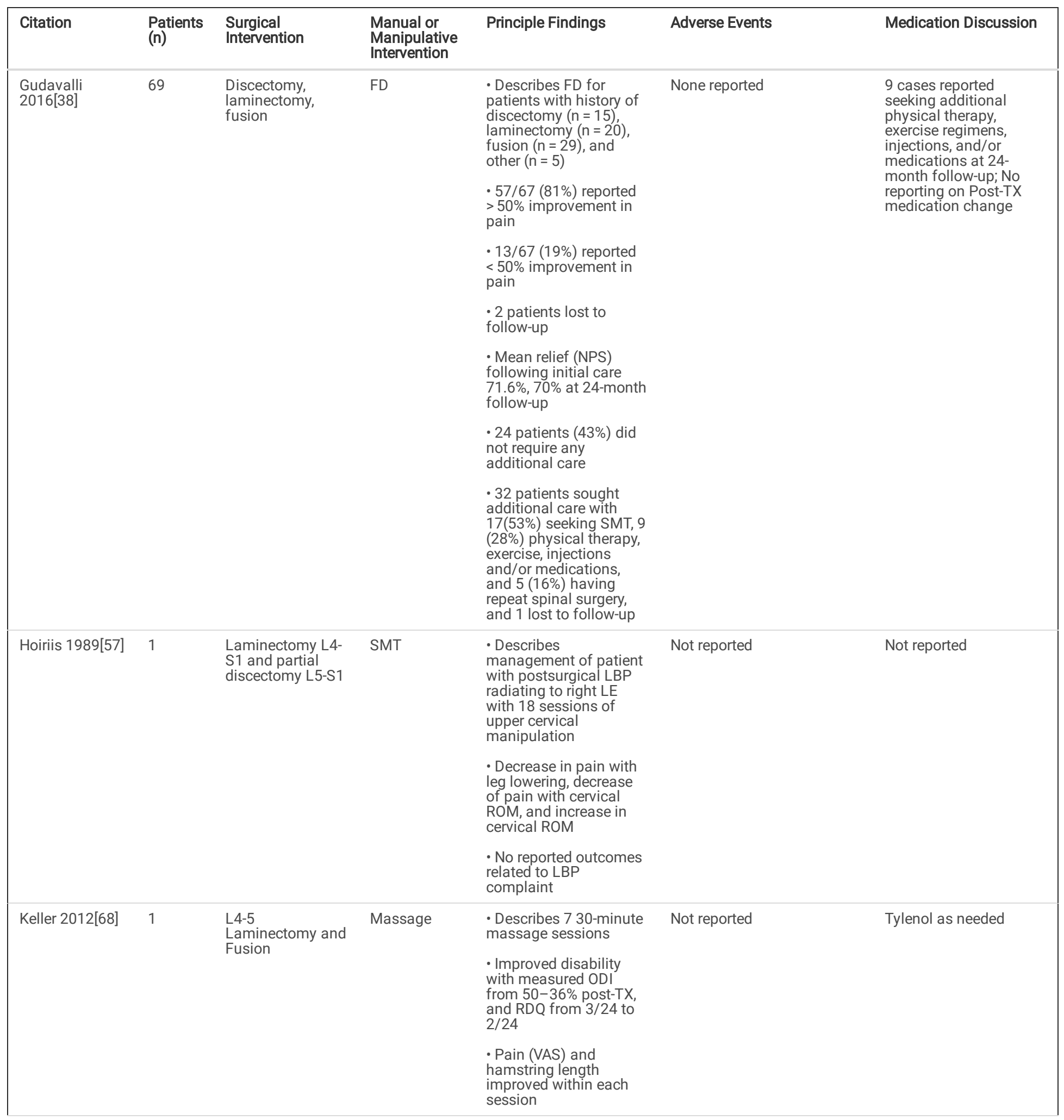

Abbreviations: ADL, activity of daily living; bid, bis in die (two times per day); cLBP, chronic low back pain; DTR, deep tendon reflexes; ESI, epidural steroid injection; FABQ, Fear-Avoidance Belief Questionnaire; FBSS, failed back surgery syndrome; FD, flexion distraction technique; FRI, Functional Rating Index; LBP, low back pain; LE, lower extremity; MUA, manipulation under anesthesia; NPS, numerical pain scale; NRS, numerical rating scale; NSAID, non-steroidal anti-inflammatory drug; ODI, Oswestry Disability Index; OMT, osteopathic manipulative therapy; OTC, over-the-counter; ROM, range of motion; RDQ, RolandMorris Disability Questionnaire; SCS, spinal cord stimulator; SMT, spinal manipulative therapy (Grade V Maitland); TX, treatment 


\begin{tabular}{|c|c|c|c|c|c|c|}
\hline Citation & $\begin{array}{l}\text { Patients } \\
\text { (n) }\end{array}$ & $\begin{array}{l}\text { Surgical } \\
\text { Intervention }\end{array}$ & $\begin{array}{l}\text { Manual or } \\
\text { Manipulative } \\
\text { Intervention }\end{array}$ & Principle Findings & Adverse Events & Medication Discussion \\
\hline \multirow[t]{5}{*}{$\begin{array}{l}\text { Kennedy } \\
2016[67]\end{array}$} & \multirow[t]{5}{*}{1} & \multirow[t]{5}{*}{$\begin{array}{l}\text { Lumbosacral } \\
\text { fusion }\end{array}$} & \multirow[t]{5}{*}{$\begin{array}{l}\text { Curanderismo } \\
\text { (massage) }\end{array}$} & $\begin{array}{l}\text { - Describes holistic } \\
\text { healing tradition } \\
\text { indigenous to Latin } \\
\text { America }\end{array}$ & \multirow[t]{5}{*}{ None reported } & \multirow{5}{*}{$\begin{array}{l}\text { Lisinopril/ hydrochloro- } \\
\text { thiazide, zolpidem, } \\
\text { clonazepam, diclofenac } \\
75 \text { mg bid, and } \\
\text { oxycodone/ } \\
\text { acetaminophen } 5 \mathrm{mg} / \\
325 \mathrm{mg} \text { bid; patient } \\
\text { reported reduced need } \\
\text { for opioid pain } \\
\text { medication }\end{array}$} \\
\hline & & & & $\begin{array}{l}\text { - Treatment consisting } \\
\text { of educating patient on } \\
\text { connection between } \\
\text { mind, body, spirit, } \\
\text { aromatherapy, music } \\
\text { therapy, and massage } \\
\text { of body meridian lines }\end{array}$ & & \\
\hline & & & & $\begin{array}{l}\text { - No quantitative } \\
\text { decrease in maximum } \\
\text { or average pain levels }\end{array}$ & & \\
\hline & & & & $\begin{array}{l}\text { - Patient reported } \\
\text { improved function, } \\
\text { mood, sleep and } \\
\text { narcotic use }\end{array}$ & & \\
\hline & & & & $\begin{array}{l}\text { - Patient did not refill } \\
\text { Percocet prescription }\end{array}$ & & \\
\hline \multirow[t]{5}{*}{ Kruse 201[53] } & \multirow[t]{5}{*}{1} & \multirow[t]{5}{*}{ Fusion } & \multirow[t]{5}{*}{ FD } & $\begin{array}{l}\text { - Describes successful } \\
\text { management of acute } \\
\text { postsurgical LBP }\end{array}$ & \multirow[t]{5}{*}{ None reported } & \multirow[t]{5}{*}{$\begin{array}{l}\text { OTC anti-inflammatory; } \\
\text { no reporting post-TX }\end{array}$} \\
\hline & & & & $\begin{array}{l}\text { - } 13 \text { sessions FD plus } \\
\text { ultrasound and } \\
\text { electrical stim over } 6 \\
\text { weeks }\end{array}$ & & \\
\hline & & & & $\begin{array}{l}\text { - Resolution of pain, } \\
\text { VAS } 5 / 10 \text { to } 0 / 10\end{array}$ & & \\
\hline & & & & $\begin{array}{l}\text { - Reduced disability, ODI } \\
18-2 \%\end{array}$ & & \\
\hline & & & & $\begin{array}{l}\text { - 2-year follow-up with } \\
\text { no symptoms } \\
\text { recurrence and } \\
\text { expressed patient } \\
\text { satisfaction with care }\end{array}$ & & \\
\hline \multirow[t]{5}{*}{ Kruse 2011[41] } & \multirow[t]{5}{*}{32} & \multirow[t]{5}{*}{$\begin{array}{l}\text { Discectomy, } \\
\text { laminectomy, } \\
\text { fusion, or } \\
\text { combination }\end{array}$} & \multirow[t]{5}{*}{ FD } & $\begin{array}{l}\text { - Retrospective analysis } \\
\text { describes FD for } \\
\text { patients with history of } \\
\text { discectomy }(n=13) \text {, } \\
\text { laminectomy }(n=10) \text {, } \\
\text { fusion }(n=2) \text {, or } \\
\text { combination }(n=7)\end{array}$ & \multirow[t]{5}{*}{ None reported } & \multirow[t]{5}{*}{ Not reported } \\
\hline & & & & - Heterogeneous sample & & \\
\hline & & & & $\begin{array}{l}\cdot \text { TX dose ranged from } \\
6-31 \text { sessions }\end{array}$ & & \\
\hline & & & & $\begin{array}{l}\text { - NPS decrease ranged } \\
\text { from 0-8.4 }\end{array}$ & & \\
\hline & & & & $\begin{array}{l}\text { - Patients with } \\
\text { combination }\end{array}$ & & \\
\hline \multirow[t]{2}{*}{ Lamb 1997[59] } & \multirow[t]{2}{*}{1} & \multirow[t]{2}{*}{ Discectomy } & \multirow[t]{2}{*}{ SMT } & $\begin{array}{l}\text { - Describes successful } \\
\text { management of patient } \\
\text { with post-surgical LBP }\end{array}$ & \multirow[t]{2}{*}{ None reported } & \multirow[t]{2}{*}{ Not reported } \\
\hline & & & & $\begin{array}{l}\text { - } 10 \text { sessions of SMT } \\
\text { (targeting sacroiliac } \\
\text { joint) and ultrasound }\end{array}$ & & \\
\hline
\end{tabular}

Abbreviations: ADL, activity of daily living; bid, bis in die (two times per day); cLBP, chronic low back pain; DTR, deep tendon reflexes; ESI, epidural steroid injection; FABQ, Fear-Avoidance Belief Questionnaire; FBSS, failed back surgery syndrome; FD, flexion distraction technique; FRI, Functional Rating Index; LBP, low back pain; LE, lower extremity; MUA, manipulation under anesthesia; NPS, numerical pain scale; NRS, numerical rating scale; NSAID, non-steroidal anti-inflammatory drug; ODI, Oswestry Disability Index; OMT, osteopathic manipulative therapy; OTC, over-the-counter; ROM, range of motion; RDQ, RolandMorris Disability Questionnaire; SCS, spinal cord stimulator; SMT, spinal manipulative therapy (Grade V Maitland); TX, treatment 


\begin{tabular}{|c|c|c|c|c|c|c|}
\hline Citation & $\begin{array}{l}\text { Patients } \\
\text { (n) }\end{array}$ & $\begin{array}{l}\text { Surgical } \\
\text { Intervention }\end{array}$ & $\begin{array}{l}\text { Manual or } \\
\text { Manipulative } \\
\text { Intervention }\end{array}$ & Principle Findings & Adverse Events & Medication Discussion \\
\hline \multirow[t]{3}{*}{$\begin{array}{l}\text { Layton } \\
2009[49]\end{array}$} & \multirow[t]{3}{*}{1} & \multirow[t]{3}{*}{ Laminectomy } & \multirow[t]{3}{*}{ SMT } & $\begin{array}{l}\text { - Describes } \\
\text { management of post- } \\
\text { surgical LBP }\end{array}$ & \multirow[t]{3}{*}{ None reported } & \multirow[t]{3}{*}{ Not reported } \\
\hline & & & & $\begin{array}{l}\text { - } 32 \text { visits of SMT of } \\
\text { cervical, thoracic, } \\
\text { lumbar and sacroiliac } \\
\text { regions }\end{array}$ & & \\
\hline & & & & $\begin{array}{l}\text { - Pain (VAS) score } \\
\text { improved from } 5 \text { to } 8, \\
\text { but Borg pain scale } \\
\text { (right now, } \\
\text { typical/average, worst) } \\
\text { was unchanged } 5,5,9 \text { to } \\
6,6,8\end{array}$ & & \\
\hline \multirow[t]{4}{*}{ Lisi 2004[50] } & \multirow[t]{4}{*}{1} & \multirow[t]{4}{*}{ Laminectomy } & \multirow[t]{4}{*}{ SMT } & $\begin{array}{l}\text { - Describes } \\
\text { management of patient } \\
\text { with residual cauda } \\
\text { equina symptoms } \\
\text { following surgical } \\
\text { decompression }\end{array}$ & \multirow[t]{4}{*}{ None reported } & \multirow[t]{4}{*}{ Not reported } \\
\hline & & & & $\begin{array}{l}\text { - Resolution of LBP } \\
\text { after } 4 \text { sessions of SMT }\end{array}$ & & \\
\hline & & & & - NRS $5 / 10$ to $0 / 10$ & & \\
\hline & & & & $\begin{array}{l}\text { - No change in chronic } \\
\text { residual cauda equina } \\
\text { symptoms }\end{array}$ & & \\
\hline \multirow[t]{7}{*}{ Lee 2017[42] } & \multirow[t]{7}{*}{102} & \multirow[t]{7}{*}{$\begin{array}{l}\text { Discectomy, } \\
\text { laminectomy, } \\
\text { fusion, or } \\
\text { combination }\end{array}$} & \multirow[t]{7}{*}{$\begin{array}{l}\text { SMT } \\
\text { (Chuna } \\
\text { Manual } \\
\text { therapy, form } \\
\text { of Korean } \\
\text { SMT) }\end{array}$} & $\begin{array}{l}\text { - Describes } \\
\text { management of } \\
\text { patients with post- } \\
\text { surgical back pain or LE } \\
\text { (spinal) pain including } \\
\text { laminectomy }(n=99) \\
\text { and/or fusion }(n=9)\end{array}$ & \multirow[t]{7}{*}{$\begin{array}{l}1 \text { case increased LBP, } 32 \\
\text { cases mild GI issues (related } \\
\text { to herbal medicine } \\
\text { component) }\end{array}$} & \multirow[t]{7}{*}{$\begin{array}{l}\text { Analgesics and muscle } \\
\text { relaxants; no reporting } \\
\text { post-TX }\end{array}$} \\
\hline & & & & $\begin{array}{l}\text { - Treatment consisted } \\
\text { of } 16 \text { weekly sessions } \\
\text { of Chuna manual } \\
\text { therapy (Korean SMT), } \\
\text { bee venom, } \\
\text { acupuncture, and herbal } \\
\text { supplementation }\end{array}$ & & \\
\hline & & & & $\begin{array}{l}\text { - } 102 \text { completed 1-year } \\
\text { follow-up }\end{array}$ & & \\
\hline & & & & $\begin{array}{l}\text { - LBP (VAS) improved } \\
\text { from } 6.1 \text { to } 2.9\end{array}$ & & \\
\hline & & & & $\begin{array}{l}\text { - LE pain (VAS) } \\
\text { improved from } 5.4 \text { to } \\
2.5\end{array}$ & & \\
\hline & & & & $\begin{array}{l}\text { - Disability (ODI) } \\
\text { reduced from } 41.3 \text { to } \\
23.6 \text { at } 6 \text {-months }\end{array}$ & & \\
\hline & & & & $\begin{array}{l}\text { - } 79.2 \% \text { sustained } \\
\text { improvement at } 1 \text {-year }\end{array}$ & & \\
\hline
\end{tabular}

Abbreviations: ADL, activity of daily living; bid, bis in die (two times per day); cLBP, chronic low back pain; DTR, deep tendon reflexes; ESI, epidural steroid injection; FABQ, Fear-Avoidance Belief Questionnaire; FBSS, failed back surgery syndrome; FD, flexion distraction technique; FRI, Functional Rating Index; LBP, low back pain; LE, lower extremity; MUA, manipulation under anesthesia; NPS, numerical pain scale; NRS, numerical rating scale; NSAID, non-steroidal anti-inflammatory drug; ODI, Oswestry Disability Index; OMT, osteopathic manipulative therapy; OTC, over-the-counter; ROM, range of motion; RDQ, RolandMorris Disability Questionnaire; SCS, spinal cord stimulator; SMT, spinal manipulative therapy (Grade V Maitland); TX, treatment 


\begin{tabular}{|c|c|c|c|c|c|c|}
\hline Citation & $\begin{array}{l}\text { Patients } \\
\text { (n) }\end{array}$ & $\begin{array}{l}\text { Surgical } \\
\text { Intervention }\end{array}$ & $\begin{array}{l}\text { Manual or } \\
\text { Manipulative } \\
\text { Intervention }\end{array}$ & Principle Findings & Adverse Events & Medication Discussion \\
\hline $\begin{array}{l}\text { Maddalozzo } \\
\text { 2018[47] }\end{array}$ & 1 & $\begin{array}{l}\text { Discectomy, } \\
\text { Fusion, } \\
\text { Hemilaminotomy }\end{array}$ & SMT & $\begin{array}{l}\text { - Describes successful } \\
\text { management of post- } \\
\text { surgical LBP } \\
\text { - Treatment consisted } \\
\text { of } 52 \text { visits over } 8 \\
\text { months with SMT with } \\
\text { active rehabilitation } \\
\text { (with functional } \\
\text { decompression) } \\
\text { - Pain (NRS) reduced } \\
\text { from } 8 / 10 \text { to } 1 / 10 \\
\text { - Disability (ODI) } \\
\text { reduced from } 50-8 \%\end{array}$ & None reported & $\begin{array}{l}\text { Hydrocodone- } \\
\text { acetominaphen } 10 / 325 \\
\text { Fentanyl } 50 \mathrm{mcg} / \mathrm{hr} \\
\text { Transdermal Patch; } \\
\text { pain medication use } \\
\text { decreased through } \\
\text { course of tx; } 41 \text {-month } \\
\text { follow-up patient denied } \\
\text { use of medication for } \\
\text { LBP }\end{array}$ \\
\hline $\begin{array}{l}\text { McGregor } \\
1983[44]\end{array}$ & 3 & $\begin{array}{l}\text { Case } 1 \text { L5/S1 } \\
\text { fusion; Case } 2 \\
\text { laminectomy; } \\
\text { Case } 3 \text { L4-S1 } \\
\text { laminectomy }\end{array}$ & SMT & $\begin{array}{l}\text { - Describes } \\
\text { management of lumbar } \\
\text { post-surgical sacroiliac } \\
\text { joint syndrome } \\
\text { - Case } 1 \text { reported } \\
\text { significant relief } \\
\text { following SMT to } \\
\text { sacroiliac joint daily for } \\
2 \text { weeks followed by } \\
\text { "regular follow-up" for } 1 \\
\text { month } \\
\text { - Case } 2 \text { reported SMT } \\
\text { to sacroiliac joint daily } \\
\text { for } 3 \text { weeks, then } \\
\text { "frequently" for a month } \\
\text { and a half, tapering over } \\
10 \text { months until no } \\
\text { longer symptomatic } \\
\text { - Case } 3 \text { describes } \\
\text { sacroiliac SMT for } 2 \\
\text { weeks with leg pain } \\
\text { completely relieved }\end{array}$ & None reported & Not reported \\
\hline $\begin{array}{l}\text { Morningstar[43] } \\
2012\end{array}$ & 3 & $\begin{array}{l}\text { Fusion and L4 or } \\
\text { L5 laminectomy }\end{array}$ & $\begin{array}{l}\text { MUA, } \\
\text { myofascial } \\
\text { trigger point } \\
\text { therapy, } \\
\text { massage }\end{array}$ & $\begin{array}{l}\text { - Describes successful } \\
\text { management of } 3 \text { cases } \\
\text { of FBSS } \\
\text { - Case } 1 \text { reduced pain } \\
\text { (NPRS) } 77 \text { to } 53 \text {, and } \\
\text { improved function (FRI) } \\
\text { from } 31 \text { to } 22 \\
\text { - Case } 2 \text { reduced pain } \\
\text { (NPRS) } 67 \text { to } 43 \text {, and } \\
\text { improved function (FRI) } \\
\text { from } 26 \text { to } 18 \\
\text { - Case } 3 \text { reduced pain } \\
\text { (NPRS) } 53 \text { to } 27 \text {, and } \\
\text { improved function (FRI) } \\
\text { from } 19 \text { to } 7\end{array}$ & 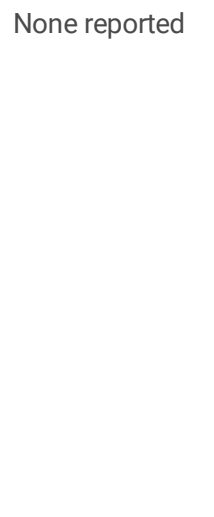 & $\begin{array}{l}\text { Case } 1: 2 \text { Vicodin } \\
7.5 / 750 \mathrm{mg} \text { no } \\
\text { reporting post-TX }\end{array}$ \\
\hline
\end{tabular}

Abbreviations: ADL, activity of daily living; bid, bis in die (two times per day); cLBP, chronic low back pain; DTR, deep tendon reflexes; ESI, epidural steroid injection; FABQ, Fear-Avoidance Belief Questionnaire; FBSS, failed back surgery syndrome; FD, flexion distraction technique; FRI, Functional Rating Index; LBP, low back pain; LE, lower extremity; MUA, manipulation under anesthesia; NPS, numerical pain scale; NRS, numerical rating scale; NSAID, non-steroidal anti-inflammatory drug; ODI, Oswestry Disability Index; OMT, osteopathic manipulative therapy; OTC, over-the-counter; ROM, range of motion; RDQ, RolandMorris Disability Questionnaire; SCS, spinal cord stimulator; SMT, spinal manipulative therapy (Grade V Maitland); TX, treatment 


\begin{tabular}{|c|c|c|c|c|c|c|}
\hline Citation & $\begin{array}{l}\text { Patients } \\
\text { (n) }\end{array}$ & $\begin{array}{l}\text { Surgical } \\
\text { Intervention }\end{array}$ & $\begin{array}{l}\text { Manual or } \\
\text { Manipulative } \\
\text { Intervention }\end{array}$ & Principle Findings & Adverse Events & Medication Discussion \\
\hline \multirow[t]{4}{*}{$\begin{array}{l}\text { Oakley } \\
2007[48]\end{array}$} & \multirow[t]{4}{*}{1} & \multirow[t]{4}{*}{ L4-5 laminectomy } & \multirow[t]{4}{*}{$\begin{array}{l}\text { SMT and } \\
\text { static } \\
\text { posturing }\end{array}$} & $\begin{array}{l}\text { - Describes successful } \\
\text { management of patient } \\
6 \text {-months post } \\
\text { laminectomy with LBP } \\
\text { and LE pain }\end{array}$ & \multirow[t]{4}{*}{ None reported } & \multirow[t]{4}{*}{$\begin{array}{l}\text { Vicodin; patient no } \\
\text { longer required } \\
\text { analgesic narcotic pain } \\
\text { medications }\end{array}$} \\
\hline & & & & $\begin{array}{l}\text { - Initial treatment } \\
\text { consisted of } 36 \text { visits } \\
\text { over } 12 \text { weeks with } \\
\text { SMT and static } \\
\text { posturing }\end{array}$ & & \\
\hline & & & & $\begin{array}{l}\text { - Pain (NRS) reduced } \\
8 / 10 \text { to } 2 / 10 \text {, disability } \\
\text { (ODI) reduced } 76-40 \% \text {, } \\
\text { repeat radiographs } \\
\text { reported improved } \\
\text { cervical lordosis }\end{array}$ & & \\
\hline & & & & $\begin{array}{l}\text { - Following additional } \\
72 \text { treatments reported } \\
\text { pain (NRS) 0/10, } \\
\text { disability (ODI) 24\%, } \\
\text { and normal ROM }\end{array}$ & & \\
\hline \multirow[t]{5}{*}{$\begin{array}{l}\text { O'Shaughnessy } \\
\text { 2010[39] }\end{array}$} & \multirow[t]{5}{*}{8} & \multirow[t]{5}{*}{$\begin{array}{l}\text { Total disc } \\
\text { replacement } \\
\text { L5/S1 (7) and/or } \\
\text { L4/L5 (4) }\end{array}$} & \multirow[t]{5}{*}{ SMT } & $\begin{array}{l}\text { - Total disc replacement } \\
\text { determined stable by } \\
\text { radiographs at } 8 \text { weeks } \\
\text { and lateral flexion- } \\
\text { extension radiographs } \\
\text { at } 12 \text { weeks }\end{array}$ & \multirow{5}{*}{$\begin{array}{l}\text { Slight increase in LBP }<12 \\
\text { hours following almost half } \\
\text { of TX; } 2 \text { patients reported } \\
\text { severe LBP and LE pain after } \\
\text { first TX; light to moderate } \\
\text { soreness common post-TX; } \\
\text { for } 5 / 8 \text { LE paresthesia } \\
\text { exacerbated for } 24-48 \\
\text { hours post-TX }\end{array}$} & \multirow[t]{5}{*}{ Not reported } \\
\hline & & & & $\begin{array}{l}\text { - Preload in sidelying } \\
\text { was performed to } \\
\text { ensure tolerance and if } \\
\text { tolerated received } \\
2 x / \text { week for } 8-10 \text { visits }\end{array}$ & & \\
\hline & & & & $\begin{array}{l}\text { - Disability }(\mathrm{ODI}) \\
\text { reduced in } 6 / 8 \text { patients }\end{array}$ & & \\
\hline & & & & $\begin{array}{l}\text { - FABQ I reduced in } 4 / 8 \\
\text { patients }\end{array}$ & & \\
\hline & & & & $\begin{array}{l}\text { - FABQ II reduced in } 5 / 8 \\
\text { patients }\end{array}$ & & \\
\hline \multirow[t]{4}{*}{ Paris 2017[62] } & \multirow[t]{4}{*}{1} & \multirow[t]{4}{*}{$\begin{array}{l}\text { T12/L2 fusion } \\
\text { post-trauma }\end{array}$} & \multirow{4}{*}{$\begin{array}{l}\text { SMT, drop } \\
\text { table assisted } \\
\text { SMT, spinal } \\
\text { mobilization }\end{array}$} & $\begin{array}{l}\text { - Describes successful } \\
\text { management with SMT }\end{array}$ & \multirow[t]{4}{*}{ None reported } & \multirow[t]{4}{*}{ Not reported } \\
\hline & & & & $\begin{array}{l}\text { - } 13 \text { sessions over } 4 \\
\text { months }\end{array}$ & & \\
\hline & & & & $\begin{array}{l}\text { - Patient self-discharged } \\
\text { and missed re- } \\
\text { examination }\end{array}$ & & \\
\hline & & & & $\begin{array}{l}\text { - Phone follow-up } \\
\text { patient indicated he felt } \\
\text { great and didn't need } \\
\text { ongoing care }\end{array}$ & & \\
\hline
\end{tabular}

Abbreviations: ADL, activity of daily living; bid, bis in die (two times per day); cLBP, chronic low back pain; DTR, deep tendon reflexes; ESI, epidural steroid injection; FABQ, Fear-Avoidance Belief Questionnaire; FBSS, failed back surgery syndrome; FD, flexion distraction technique; FRI, Functional Rating Index; LBP, low back pain; LE, lower extremity; MUA, manipulation under anesthesia; NPS, numerical pain scale; NRS, numerical rating scale; NSAID, non-steroidal anti-inflammatory drug; ODI, Oswestry Disability Index; OMT, osteopathic manipulative therapy; OTC, over-the-counter; ROM, range of motion; RDQ, RolandMorris Disability Questionnaire; SCS, spinal cord stimulator; SMT, spinal manipulative therapy (Grade V Maitland); TX, treatment 


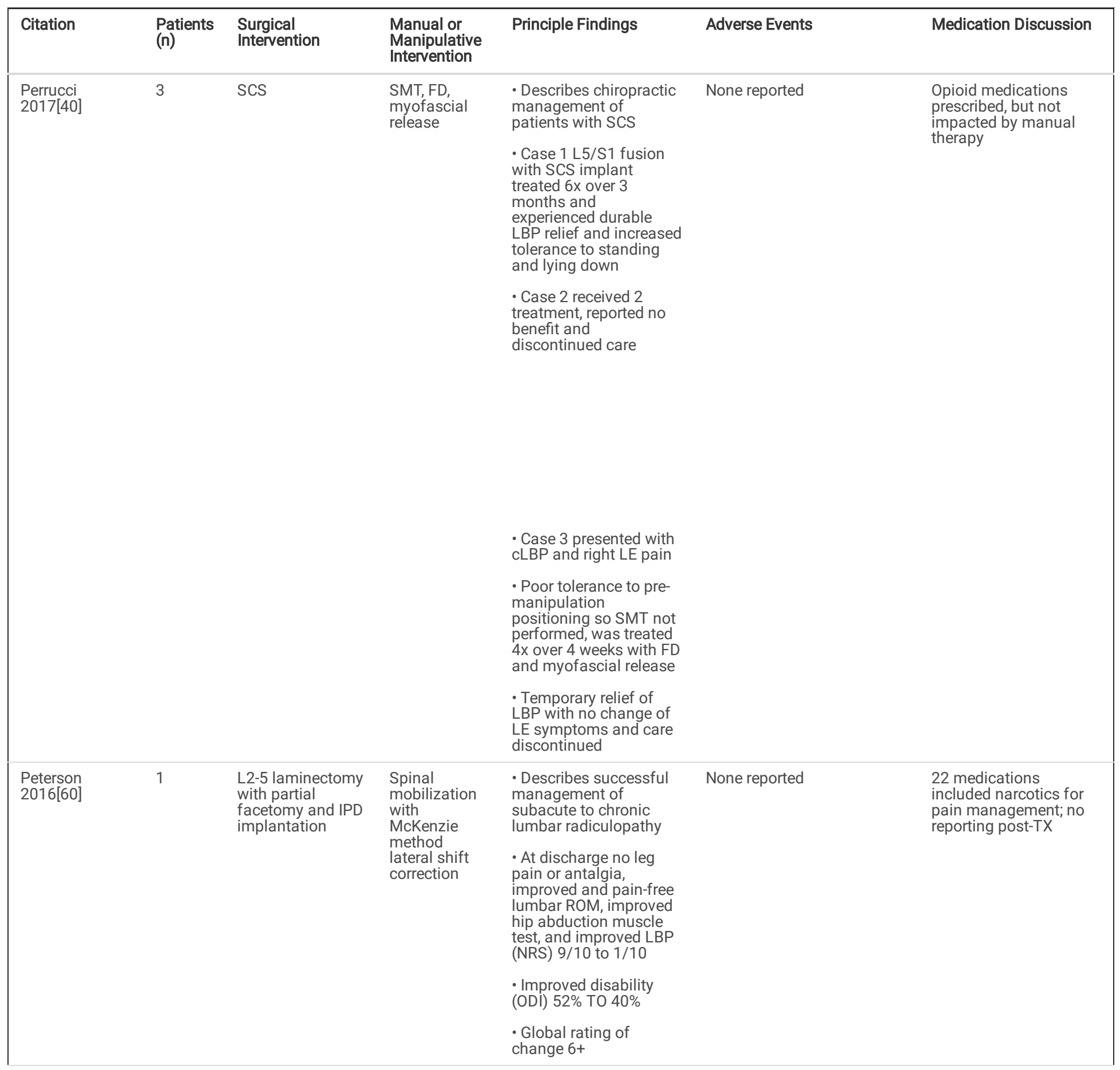

Abbreviations: ADL, activity of daily living; bid, bis in die (two times per day); cLBP, chronic low back pain; DTR, deep tendon reflexes; ESI, epidural steroid injection; FABQ, Fear-Avoidance Belief Questionnaire; FBSS, failed back surgery syndrome; FD, flexion distraction technique; FRI, Functional Rating Index; LBP, low back pain; LE, lower extremity; MUA, manipulation under anesthesia; NPS, numerical pain scale; NRS, numerical rating scale; NSAID, non-steroidal anti-inflammatory drug; ODI, Oswestry Disability Index; OMT, osteopathic manipulative therapy; OTC, over-the-counter; ROM, range of motion; RDQ, RolandMorris Disability Questionnaire; SCS, spinal cord stimulator; SMT, spinal manipulative therapy (Grade V Maitland); TX, treatment 


\begin{tabular}{|c|c|c|c|c|c|c|}
\hline Citation & $\begin{array}{l}\text { Patients } \\
\text { (n) }\end{array}$ & $\begin{array}{l}\text { Surgical } \\
\text { Intervention }\end{array}$ & $\begin{array}{l}\text { Manual or } \\
\text { Manipulative } \\
\text { Intervention }\end{array}$ & Principle Findings & Adverse Events & Medication Discussion \\
\hline \multirow[t]{3}{*}{ Stern 1995[46] } & 7 & Undifferentiated & $\begin{array}{l}\text { SMT, } \\
\text { massage, } \\
\text { mobilization }\end{array}$ & $\begin{array}{l}\text { - Case series of } 3531 \\
\text { patient files with } n=71 \\
\text { having LBP and LE pain } \\
\text { with diagnosis of disc } \\
\text { herniation, of those } 7 \\
\text { had history of low back } \\
\text { surgery }\end{array}$ & None reported & Not reported \\
\hline & & & & $\begin{array}{l}\text { - History of lumbar } \\
\text { surgery more common } \\
\text { in negative (non- } \\
\text { response) outcome } \\
\text { group }(p=0.007)\end{array}$ & & \\
\hline & & & & $\begin{array}{l}\text { - Previous operation } \\
\text { tended to predict poor } \\
\text { outcome: adjusted odds } \\
\text { ratio } 46.6(\mathrm{Cl} 2.4-90.0)\end{array}$ & & \\
\hline \multirow[t]{3}{*}{ Shaw 1996[64] } & 1 & $\begin{array}{l}\text { L4-5 discectomy } \\
\text { and laminectomy }\end{array}$ & SMT & $\begin{array}{l}\text { - Describes response to } \\
\text { new LBP with right S1 } \\
\text { radicular pain after slip } \\
\text { and fall with prior low } \\
\text { back surgery }\end{array}$ & None reported & $\begin{array}{l}\text { Prozac and Advil; no } \\
\text { reporting post-TX }\end{array}$ \\
\hline & & & & $\begin{array}{l}\text { - Reduced disability } \\
\text { (ODI) from } 84 \% \text { to }< \\
10 \%\end{array}$ & & \\
\hline & & & & $\begin{array}{l}\text { - Treatment consisted } \\
\text { of SMT, passive } \\
\text { physiotherapy, and } \\
\text { active and passive } \\
\text { home care with } \\
\text { definitive treatment } \\
\text { dosage described }\end{array}$ & & \\
\hline \multirow[t]{4}{*}{ Taylor 2007[66] } & 1 & $\begin{array}{l}\text { L4-5 } \\
\text { decompression } \\
\text { with laminectomy } \\
\text { and cyst excision }\end{array}$ & FD & $\begin{array}{l}\text { - Describes care of } \\
\text { patient with LBP and } \\
\text { bilateral LE symptoms, } \\
\text { and similar symptoms } \\
\text { resolved } 3 \text {-years prior } \\
\text { with surgery }\end{array}$ & None reported & Not reported \\
\hline & & & & $\begin{array}{l}\text { - Treatment with FD } \\
\text { provided limited relief } \\
\text { and updated MRI } \\
\text { revealed L4-5 synovial } \\
\text { cyst with progression of } \\
\text { Grade } 1 \text { L4 } \\
\text { spondylolisthesis }\end{array}$ & & \\
\hline & & & & $\begin{array}{l}\text { - Lumbar stability } \\
\text { exercise initiated with } \\
\text { palliative effect and } \\
\text { patient progressed to } \\
\text { self-management }\end{array}$ & & \\
\hline & & & & $\begin{array}{l}\text { - Disability (ODI) } \\
\text { reduced from } 30-12.5 \% \\
\text { at } 2.5 \text {-year follow-up }\end{array}$ & & \\
\hline \multirow[t]{3}{*}{$\begin{array}{l}\text { Vaillancourt } \\
\text { 1983[69] }\end{array}$} & 1 & L4-S1 fusion & SMT & $\begin{array}{l}\text { - Describes of patient } \\
\text { with cLBP, bilateral LE } \\
\text { pain and L4 } \\
\text { hypoesthesia }\end{array}$ & None reported & $\begin{array}{l}\text { Switched from } \\
\text { Carbamazepine to } \\
\text { Aspirin at Psychiatrist } \\
\text { direction }\end{array}$ \\
\hline & & & & $\begin{array}{l}\text { - Treatment consisted } \\
\text { of } 14 \text { upper cervical } \\
\text { manipulations over } 166 \\
\text { days }\end{array}$ & & \\
\hline & & & & $\begin{array}{l}\text { - No valid outcomes } \\
\text { were available other } \\
\text { than reported LE pain } \\
\text { reduction and } \\
\text { medication reduction }\end{array}$ & & \\
\hline
\end{tabular}

Abbreviations: ADL, activity of daily living; bid, bis in die (two times per day); cLBP, chronic low back pain; DTR, deep tendon reflexes; ESI, epidural steroid injection; FABQ, Fear-Avoidance Belief Questionnaire; FBSS, failed back surgery syndrome; FD, flexion distraction technique; FRI, Functional Rating Index; LBP, low back pain; LE, lower extremity; MUA, manipulation under anesthesia; NPS, numerical pain scale; NRS, numerical rating scale; NSAID, non-steroidal anti-inflammatory drug; ODI, Oswestry Disability Index; OMT, osteopathic manipulative therapy; OTC, over-the-counter; ROM, range of motion; RDQ, RolandMorris Disability Questionnaire; SCS, spinal cord stimulator; SMT, spinal manipulative therapy (Grade V Maitland); TX, treatment 


\begin{tabular}{|c|c|c|c|c|c|c|}
\hline Citation & $\begin{array}{l}\text { Patients } \\
\text { (n) }\end{array}$ & $\begin{array}{l}\text { Surgical } \\
\text { Intervention }\end{array}$ & $\begin{array}{l}\text { Manual or } \\
\text { Manipulative } \\
\text { Intervention }\end{array}$ & Principle Findings & Adverse Events & Medication Discussion \\
\hline \multirow[t]{2}{*}{$\begin{array}{l}\text { Vulfsons } \\
\text { 2011[63] }\end{array}$} & \multirow[t]{2}{*}{1} & \multirow[t]{2}{*}{$\begin{array}{l}\text { Hemilaminectomy } \\
\text { and discectomy } \\
\text { with revision } 2 x\end{array}$} & \multirow[t]{2}{*}{$\begin{array}{l}\text { OMT } \\
\text { (oscillatory) }\end{array}$} & $\begin{array}{l}\text { - Returned to work as a } \\
\text { surgeon at } 4 \text {-month } \\
\text { follow-up }\end{array}$ & \multirow[t]{2}{*}{ None reported } & \multirow[t]{2}{*}{ Not reported } \\
\hline & & & & - Without pain & & \\
\hline \multirow[t]{5}{*}{ Welk 2012[52] } & \multirow[t]{5}{*}{1} & \multirow[t]{5}{*}{$\begin{array}{l}\text { Lumbar } \\
\text { discectomy }\end{array}$} & \multirow[t]{5}{*}{$\begin{array}{l}\text { FD, manual } \\
\text { therapy }\end{array}$} & $\begin{array}{l}\text { - Describes } \\
\text { management of acute } \\
\text { on chronic LBP with } \\
\text { right gluteal pain }\end{array}$ & \multirow[t]{5}{*}{ None reported } & \multirow[t]{5}{*}{$\begin{array}{l}\text { Flexeril, Naprosyn, } \\
\text { Percocet as needed; no } \\
\text { reporting Post-TX }\end{array}$} \\
\hline & & & & $\begin{array}{l}\text { - MRI revealed recurrent } \\
\text { L5/S1 disc herniation } \\
\text { and epidural fibrosis } \\
\text { and patient declined } \\
\text { surgical revision }\end{array}$ & & \\
\hline & & & & $\begin{array}{l}\text { - Care consisted of } 27 \\
\text { visits over } 12 \text { weeks, } \\
\text { then every other week } \\
\text { for } 9 \text { visits }\end{array}$ & & \\
\hline & & & & $\begin{array}{l}\text { - Disability }(O D I) \\
\text { reduced from } 50-17.7 \% \\
\text { in } 10 \text { weeks }\end{array}$ & & \\
\hline & & & & $\begin{array}{l}\text { - Other outcomes } \\
\text { included pain intensity, } \\
\text { orthopedic tests, lumbar } \\
\text { ROM and DTRs }\end{array}$ & & \\
\hline $\begin{array}{l}\text { Abbreviations: } \\
\text { injection; FABQ } \\
\text { LBP, low back p } \\
\text { anti-inflammat } \\
\text { Morris Disabilit }\end{array}$ & $\begin{array}{l}\text { activity o } \\
\text { r-Avoidan } \\
\text { LE, lower } \\
\text { rug; ODI, } \\
\text { estionnai }\end{array}$ & $\begin{array}{l}\text { Jaily living; bid, bis } \\
\text { Belief Questionnai } \\
\text { tremity; MUA, mani } \\
\text { westry Disability In } \\
\text { SCS, spinal cord st }\end{array}$ & $\begin{array}{l}\text { die (two times } \\
\text { FBSS, failed b } \\
\text { lation under ar } \\
\text { x; OMT, osteop } \\
\text { ulator; SMT, sp }\end{array}$ & $\begin{array}{l}\text { day); CLBP, chronic low } \\
\text { surgery syndrome; FD, } \\
\text { thesia; NPS, numerical p } \\
\text { ic manipulative therapy; } \\
\text { al manipulative therapy }\end{array}$ & $\begin{array}{l}\text { ck pain; DTR, dee } \\
\text { xion distraction te } \\
\text { n scale; NRS, num } \\
\text { TC, over-the-count } \\
\text { ade V Maitland); }\end{array}$ & $\begin{array}{l}\text { es; ESI, epidural steroid } \\
\text { unctional Rating Index; } \\
\text { ale; NSAID, non-steroidal } \\
\text { of motion; RDQ, Roland- }\end{array}$ \\
\hline
\end{tabular}

Table 13

Evidence table for consensus studies, guidelines, and commentaries

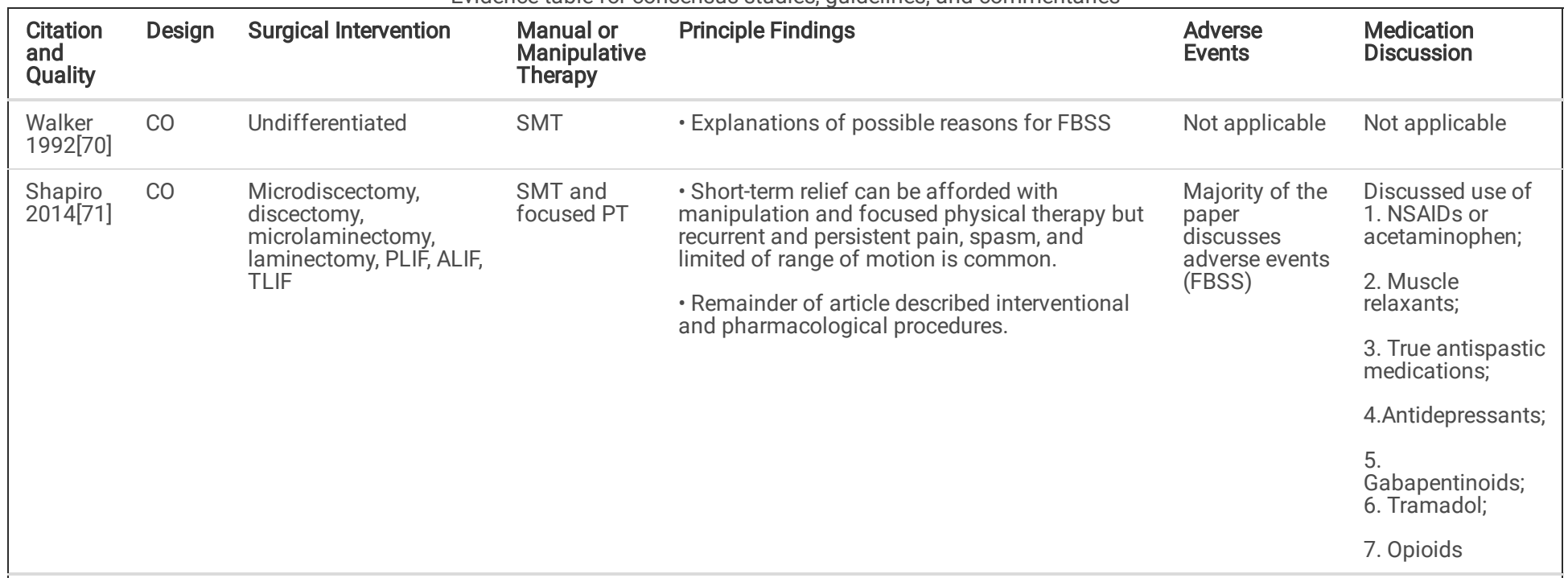

Abbreviations: ALIF, anterior lumbar interbody fusion; CO, commentary; FBSS, failed back surgery syndrome; PLIF, posterior lumbar interbody fusion; NSAID, nonsteroidal anti-inflammatory drugs; PT, physical therapy; TCA, tricyclic antidepressants; TLIF, transforaminal lumbar interbody fusion;

\section{Strength Of Evidence}

The strength of evidence is rated and grouped by prior surgical type and criteria are described in Table 6 .

\section{Discectomy}

Evidence was inconclusive because of a scarcity of studies and is insufficient to recommend or discourage application of MMT in treatment plans following lumbar discectomy.

\section{Laminectomy}


Evidence was inconclusive regarding spinal mobilization (Grade III or IV Maitland) following L5 laminectomy but is favorable for improving lumbar extension ROM without improving pain and function outcome measures. Evidence is insufficient to recommend or discourage application of MMT in treatment plans following lumbar laminectomy.

\section{Fusion}

Evidence was inconclusive because of a scarcity of studies and is insufficient to recommend or discourage application of MMT in treatment plans following lumbar fusion.

\section{Disc replacement}

Evidence was inconclusive because of a scarcity of studies and is insufficient to recommend or discourage application of MMT in treatment plans following lumbar total disc replacement.

\section{Spinal cord stimulator}

Evidence was inconclusive because of a scarcity of studies and is insufficient to recommend or discourage application of MMT in treatment plans following spinal cord stimulator implantation.

\section{Undifferentiated postsurgical (lumbar discectomy, laminectomy, or fusion)}

Moderate evidence indicates that mixed technique PT (which may include MMT) does not improve outcomes compared with control or standard PT techniques. Moderate evidence indicates adding neural mobilization to immediate postoperative care does not improve outcomes.

\section{Discussion}

This review evaluated the state of literature, assessed RCT and SR quality, and graded the strength of evidence for MMT for individuals with history of lumbar surgical procedures. We organized the findings and graded the strength of evidence by surgical type. Very few MMT clinical trials have been completed for this population and thus the interested clinician is forced to rely heavily on case reports and series for literature guidance.

\section{Discectomy}

We found no trials that specifically investigated MMT following discectomy. There were 3 pilot studies published by Kim et al. investigated OMT versus active control following microdiscectomy [72-74]. Two of these studies were of the same patient data (short- and long-term follow-up), and all 3 described greater improvements in pain and disability following OMT. There were 10 case reports and series describing the care of 54 patients following discectomy. All but one intraoperative report [34] involved care provided by chiropractors. Favorable responses were reported with spinal manipulation [33, 37, 51, 59], FD manipulation [38, 41, 51, 52], manual therapy [52, 56], and manipulation under anesthesia of the sciatic nerve [34] or spinal joints [36]. Following discectomy, a scoping review suggested early passive and active hip and knee flexion exercises to reduce time to independent mobility and return-to-work [31].

\section{Laminectomy}

One randomized controlled trial with acceptable quality met inclusion criteria. Relevant to MMT this trial found that lumbar mobilization increased lumbar extension ROM after laminectomy. Grade III and IV mobilization did not significantly improve functional measures for lower back pain. As this was the only study specific to laminectomy and it was of acceptable quality, there is inconclusive evidence for or against using MMT. We identified 16 case reports or series describing 143 patients after lumbar laminectomy. Two reports from the medical profession [42, 63], 1 from physical therapy [60], 1 from an athletic trainer [45] and the rest were chiropractic specific. Favorable responses were described with spinal manipulation $[42,44,48-50,57,64,65]$, spinal manipulation under anesthesia [35], spinal mobilization with or without McKenzie method [60], FD manipulation [38, 41, 51, 66], and massage [45].

\section{Fusion}

We found no trials that specifically investigated MMT following discectomy. We identified 16 case reports or series describing MMT for 67 patients with history of lumbar fusion. Three of these reports were from the medical profession [34, 42, 67], 1 from massage [68] and the rest were chiropractic specific. Favorable response to care was noted following spinal manipulation [42, 44, 47, 62, 69], FD manipulation [38, 41, 53-55, 58], massage [67, 68], neural mobilization both post- [61] and intra-operative [34], and spinal manipulation under anesthesia [43]. A literature review outlined types of lumbar fusion operation, common adverse events, and described chiropractic fusion related literature while calling for clinical trials to assess the safety and efficacy of care [32].

\section{Disc Replacement}

No trials and only 1 case described MMT following lumbar total disc replacement [39]. O'Shaughnessy et al. described management of 8 cases with spinal manipulation. As a safety measure, the authors incorporated flexion-extension radiographs to ensure intersegmental stability and patients were positioned in a preloaded manipulative setup to determine tolerance. Disability and fear-avoidance was improved in $75 \%(6 / 8)$ and $63 \%$ (5/8) of cases respectively.

\section{Spinal cord stimulator}

No trials and only 1 case report described MMT following spinal cord stimulator [40]. This report outlined chiropractic management of 3 cases through a combination of spinal manipulation, FD, and myofascial release. One of the patients could not tolerate positioning for spinal manipulation and as a result, was not performed. Two of the 3 cases reported favorable outcomes and one had no benefit from care.

Postsurgical undifferentiated (lumbar discectomy, laminectomy, or fusion)

Page 23/30 
Two of the 3 randomized controlled trials enrolled patients following a variety of different lumbar surgical procedures (discectomy, laminectomy, and fusion) and did not breakdown their results by surgical type [28, 29]. The studies were both early postoperative, and neither study found significant improvement by incorporating MMT. The study by Mannion et al. did not specifically require MMT as part of the intervention group [29]. In a scoping review of lumbar surgery perioperative rehabilitation, Marchand et al. found that passive and active hip and knee flexion exercises reduced time to independent mobility and return-towork. Commentaries by Walker [70] and Shapiro [71] discussed complications related to, and the role of manipulation for, individuals with FBSS.

\section{Adverse events}

None of the clinical trials reported patient dropout in any treatment groups including MMT. Each of the pilot trials reported patients lost to outcome, but no side effects or complications were reported [72-74]. None of the case reports or series reported any serious adverse events such as loss of bowel or bladder function, stroke, fracture or hospitalization [75]. The case series describing intraoperative neural mobilization reported 61\% (19/31) patients noted increased pain post MMT and 29\% (9/31) required additional exploratory surgery [34]. Mild lumbar soreness was reported by several case reports for various MMTs and surgical types [39, 42, 51]; however, mild soreness is commonly reported following manual therapy in patients without history of surgery [76-78]. One study reported increased lower extremity pain in 2 of 8 patients being treated with spinal manipulation following lumbar total disc replacement [39].

\section{Medications}

None of the adequately powered trials used pharmacologic prescription or utilization as an outcome, thus no conclusions or recommendations can be determined regarding the ability of MMT to reduce or impact patient usage of medication. One pilot trial assessed anti-inflammatory, analgesic, and muscle relaxant medication usage as a secondary outcome and found that patients assigned to osteopathic manipulative rehabilitation after microdiscectomy used less medication than the control group [72]. A few case reports similarly described patient medication reduction or elimination through the utilization of MMT $[47,48,56,67]$, but most did not comment on any change in medication. A recent systematic review and meta-analysis revealed an inverse association between chiropractic care and opioid receipt in veterans with spinal pain [79] and multiple cohort studies of health insurance claims data displayed a significantly lower likelihood of filling opioid prescriptions for recipients of chiropractic care than nonrecipients [80, 81]. Although promising, it is not clear if this relationship persists in the post-surgical population.

\section{Limitations}

This review is limited by the evidence that is available and underscores the knowledge gap and the need for high-quality trials to allow for recommendations for or against MMT following a variety of lumbar surgeries. Although numerous case reports describe favorable outcomes with MMT, the limited number of RCTs and the absence of cohort studies with comparison make it impractical to make recommendations for most MMT. There is insufficient evidence to make any recommendations following most surgical procedures. Two of the 3 sufficiently powered RCTs included multimodal care, were heterogeneous of design, and all 3 were perioperative, making the findings impossible to pool and challenging to generalize to outpatient settings with patients presenting months to years post-procedure. Further, none of these trials specifically investigated or included spinal manipulation as an intervention. The current literature to guide clinicians relies heavily on case reports, with which there is a strong prospect of positive publication bias and likely under-reporting of adverse events [82].

The increased utilization of surgical intervention to address lumbar degenerative conditions and high rate of spine pain recurrence necessitate the need for studying MMT as a non-pharmacological treatment option post-operatively. Further study is needed which emphasizes pragmatic application of MMT within study designs. RCTs and longitudinal cohorts with comparison or control groups could shed light on the relative safety or dosage of MMT that is appropriate to reach maximum therapeutic benefit. There is a need to assess the impact of MMT on prescription medication utilization. Lastly, there is a need for studies that stratify the response to MMT by surgical type. There may be between-group differences for treatments depending on surgical-type history. Although lowlevel studies suggest favorable outcomes associated with MMT in the postsurgical patient, no conclusions can be drawn from the evidence related to timing, dosage, tolerance, or safety of MMT after lumbar surgery.

\section{Conclusions}

The findings of this review will help to inform practitioners of MMT (chiropractors, physical therapists, osteopaths, massage therapists and other manual therapy providers) about existing literature for managing patients with prior lumbar surgeries. Following lumbar surgery, current evidence indicated that inpatient neural mobilization does not improve outcomes. There is inconclusive evidence to recommend for or against most MMT after most surgical interventions. The overall body of evidence is primarily limited to low-level studies including case reports and series. The results of this study suggest that MMT may have a positive effect in individuals with low back pain with a history of lumbar surgery, however, caution should be used in generalizing the findings of these results to clinical practice, considering the low-quality of the evidence available for synthesis. High-quality studies, including RCTs are needed to gain further understanding of the effectiveness and safety profile of MMT for patients with prior lumbar surgery.

\section{Abbreviations}

Flexion-distraction (FD), Manual and manipulative therapy (MMT), osteopathic manipulative therapy (OMT), randomized controlled trial (RCT), Scottish Intercollegiate Guidelines Network (SIGN), systematic review (SR)

\section{Declarations}

\section{Ethics approval and consent to participate}

Since our study is a systematic review, an ethical review is not required. 


\section{Consent for publish}

Not applicable

\section{Availability of data and materials}

Not applicable. The data used for analysis was retrieved from published studies listed in our manuscript.

\section{Competing interests}

The authors declare they have no competing interests.

\section{Funding}

There was no funding for this study.

\section{Authors' contributions}

$\mathrm{CD}$ and $\mathrm{CH}$ developed the study concept. CD, JG, ZC, SW and CH designed study methodology. $\mathrm{CD}$, ZC and SW performed literature search and data collection. $\mathrm{CD}, J \mathrm{G}, \mathrm{ZC}, \mathrm{NH}, \mathrm{DG}$, and $\mathrm{AS}$ analyzed and interpreted the data. All authors read and approved the final manuscript.

\section{Authors' Information}

1VA Puget Sound Health Care System. 2Butler VA Healthcare. 3Medical College of Wisconsin. 4Logan University. 5VA Central lowa Health Care System. 6VA Palo Alto Health Care System. 7VA Western New York Healthcare System, 8Texas Chiropractic College.

\section{Acknowledgements}

Not applicable

\section{References}

1. GBD 2017 Disease and Injury Incidence and Prevalence Collaborators. Global, regional, and national incidence, prevalence, and years lived with disability for 354 diseases and injuries for 195 countries and territories, 1990-2017: a systematic analysis for the Global Burden of Disease Study 2017. Lancet. 2018 10;392(10159):1789-1858.

2. Yoshihara $\mathrm{H}$, Yoneoka D. National trends in the surgical treatment for lumbar degenerative disc disease: United States, 2000 to 2009 . Spine J. 2015;15(2):265-71.

3. Pumberger M, Chiu Y-L, Ma Y, Girardi FP, Mazumdar M, Memtsoudis SG. National in-hospital morbidity and mortality trends after lumbar fusion surgery between 1998 and 2008. J Bone Joint Surg Br. 2012;94(3):359-64.

4. Martin BI, Mirza SK, Spina N, Spiker WR, Lawrence B, Brodke DS. Trends in Lumbar Fusion Procedure Rates and Associated Hospital Costs for Degenerative Spinal Diseases in the United States, 2004 to 2015. Spine. 2019;44(5):369-76.

5. Kha ST, llyas H, Tanenbaum JE, et al. Trends in Lumbar Fusion Surgery Among Octogenarians: A Nationwide Inpatient Sample Study From 2004 to 2013. Global Spine J -. 2018;8(6):593-9.

6. Ruan W, Feng F, Liu Z, Xie J, Cai L, Ping A. Comparison of percutaneous endoscopic lumbar discectomy versus open lumbar microdiscectomy for lumbar disc herniation: A meta-analysis. Int J Surg. 2016;31:86-92.

7. Yadav RI, Long L, Yanming C. Comparison of the effectiveness and outcome of microendoscopic and open discectomy in patients suffering from lumbar disc herniation. Med (Baltim). 2019;98(50):e16627.

8. Carragee EJ, Han MY, Suen PW, Kim D. Clinical outcomes after lumbar discectomy for sciatica: the effects of fragment type and anular competence. J Bone Joint Surg Am. 2003;85(1):102-8.

9. Maigne JY, Planchon CA. Sacroiliac joint pain after lumbar fusion. A study with anesthetic blocks. Eur Spine J. 2005;14(7):654-8.

10. Fritsch EW, Heisel J, Rupp S. The failed back surgery syndrome: reasons, intraoperative findings, and long-term results: a report of 182 operative treatments. Spine (Phila Pa 1976). 1996;21(5):626-33.

11. Suri P, Pearson AM, Zhao W, et al. Pain Recurrence After Discectomy for Symptomatic Lumbar Disc Herniation. Spine (Phila Pa 1976). 2017;42(10):75563.

12. Castillo H, Chintapalli RTV, Boyajian HH, Cruz SA, Morgan VK, Shi LL, et al. Lumbar discectomy is associated with higher rates of lumbar fusion. Spine J. 2019;19(3):487-92. 
13. Turner JA, Loeser JD, Deyo RA, Sanders SB. Spinal cord stimulation for patients with failed back surgery syndrome or complex regional pain syndrome: a systematic review of effectiveness and complications. Pain. 2004;108(1-2):137-47.

14. Kim C-G, Mun S-J, Kim K-N, Shin B-C, Kim N-K, Lee D-H, et al. Economic evaluation of manual therapy for musculoskeletal diseases: a protocol for a systematic review and narrative synthesis of evidence. BMJ Open. 2016;6(5).

15. Smith MS, Olivas J, Smith K. Manipulative Therapies: What Works. Am Fam Physician. 2019;99(4):248-52.

16. Brantingham JW, Cassa TK, Bonnefin D, Pribicevic M, Robb A, Pollard H, et al. Manipulative and multimodal therapy for upper extremity and temporomandibular disorders: a systematic review. J Manipulative Physiol Ther. 2013;36(3):143-201.

17. Murad MH, Sultan S, Haffar S, Bazerbachi F. Methodological quality and synthesis of case series and case reports. BMJ Evid-Based Med. 2018;23(2):603.

18. Crumpston M, Li T, Page MJ, et al. Updated guidance for trusted systematic reviews: a new edition of the Cochrane Handbook for Systematic Reviews of Interventions. Cochrane Database Syst Rev. 2019;10:ED000142.

19. Scherer RW, Meerpohl JJ, Pfeifer N, Schmucker C, Schwarzer G, von Elm E. Full publication of results initially presented in abstracts. Cochrane Database Syst Rev. 2018;11:MR000005.

20. Scottish Intercollegiate Guidelines Network. Critical appraisal and notes checklists. https://www.sign.ac.uk/checklists-and-notes Accessed 1 March 2020.

21. Hawk C, Minkalis AL, Khorsan R, Daniels CJ, Homack D, Gliedt JA, et al. Systematic Review of Nondrug, Nonsurgical Treatment of Shoulder Conditions. J Manipulative Physiol Ther. 2017;40(5):293-319.

22. Bronfort G, Haas M, Evans R, Leininger B, Triano J. Effectiveness of manual therapies: the UK evidence report. Chiropr Osteopat. 2010;18:3.

23. Clar C, Tsertsvadze A, Court R, Hundt GL, Clarke A, Sutcliffe P. Clinical effectiveness of manual therapy for the management of musculoskeletal and nonmusculoskeletal conditions: systematic review and update of UK evidence report. Chiropr Man Ther. 2014;22(1):12.

24. Moher D, Liberati A, Tetzlaff J, Altman DG, PRISMA Group. Preferred reporting items for systematic reviews and meta-analyses: the PRISMA statement. PLoS Med. 2009 Jul;21(7):e1000097. 6(.

25. Basson A, Olivier B, Ellis R, Coppieters M, Stewart A, Mudzi W. The Effectiveness of Neural Mobilization for Neuromusculoskeletal Conditions: A Systematic Review and Meta-analysis. J Orthop Sports Phys Ther. 2017;47(9):593-615.

26. Gilmore SJ, McClelland JA, Davison M. Physiotherapeutic interventions before and after surgery for degenerative lumbar conditions: a systematic review. Physiotherapy. 2015;101(2):111-8.

27. Madera M, Brady J, Deily S, et al. The role of physical therapy and rehabilitation after lumbar fusion surgery for degenerative disease: a systematic review. J Neurosurg Spine. 2017;26(6):694-704.

28. Scrimshaw SV, Maher CG. Randomized controlled trial of neural mobilization after spinal surgery. Spine. 2001;26(24):2647-52.

29. Mannion AF, Denzler R, Dvorak J, Müntener M, Grob D. A randomised controlled trial of post-operative rehabilitation after surgical decompression of the lumbar spine. Eur Spine J. 2007;16(8):1101-17.

30. Timm KE. A randomized-control study of active and passive treatments for chronic low back pain following L5 laminectomy. J Orthop Sports Phys Ther. 1994;20(6):276-86.

31. Marchand AA, O'Shaughnessy J, Châtillon C, et al. Practices in Lumbar Surgery Perioperative Rehabilitation: A Scoping Review. J Manipulative Physiol Ther. 2016;39(9):668-92.

32. Daniels CJ, Wakefield PJ, Bub GA, Toombs JD. A narrative review of lumbar fusion surgery with relevance to chiropractic practice. J Chiropr Med. 2016;15(4):259-71.

33. Adams V. The rehabilitation of a patient with functional instability associated with failed back surgery. J Am Chiropr Assoc. 2004;41(12):31-9.

34. Adams JE, Inman VT. Stretching of the Sciatic Nerve. California Medicine. 1959;91(1):24-6.

35. Alexander GK. Manipulation under anaesthesia of lumbar post-laminectomy syndrome patients with epidural fibrosis and recurrent HNP. J Chiropr. 1993;30(6a):79-82.

36. Aspergren DD, Hemler DE, Wright RE. Manipulation under epidural anesthesia with corticosteroid injection: two case reports. J Manipulative Physiol Ther. 1997;20:4:263-266.

37. Benningfield RC. Conservative treatment and back-strengthening exercises to prevent recurrent surgery. J Sports Chiropr Rehabil. 1997;11(2):52-6._.

38. Gudavalli MR, Olding K, Joachim G, Cox JM. Chiropractic Distraction Spinal Manipulation on Postsurgical Continued Low Back and Radicular Pain Patients: A Retrospective Case Series. J Chiropr Med. 2016;15(2):121-8.

39. O'Shaughnessy J, Drolet M, Roy J-F, Descarreaux M. Chiropractic management of patients post-disc arthroplasty: eight case reports. Chiropr Osteopat. 2010;18(1):7.

40. Perrucci RM, Coulis CM. Chiropractic management of post spinal cord stimulator spine pain: a case report. Chiropr Man Ther. 2017;25(1):5.

41. Kruse RA, Cambron J. Chiropractic Management of Postsurgical Lumbar Spine Pain: A Retrospective Study of 32 Cases. J Manipulative Physiol Ther. 2011;34(6):408-12.

42. Lee J, Shin J-S, Lee YJ, Kim M, Choi A, Lee J-H, et al. Long-Term Course of Failed Back Surgery Syndrome (FBSS) Patients Receiving Integrative Korean Medicine Treatment: A 1 Year Prospective Observational Multicenter Study. PLOS ONE. 2017;12(1):e0170972.

43. Morningstar MW, Strauchman MN. Manipulation under anesthesia for patients with failed back surgery: retrospective report of 3 cases with 1-year followup. J Chiropr Med. 2012;11(1):30-5.

44. McGregor M, Cassidy JD. Post-surgical sacroiliac joint syndrome. J Manipulative Physiol Ther. 1983;6(1):1-11.

Page 26/30 
45. Bates WT. Postoperative Disk Management in Sports. Phys Ther. 1964;44(11):997-8.

46. Stern PJ, Cote P, Cassidy JD. A series of consecutive cases of low back pain with radiating leg pain treated by chiropractors. J Manipulative Physiol Ther. 1995;18(6):335-42.

47. Maddalozzo GF, Aikenhead K, Sheth V, Perisic MN. A novel treatment combination for failed back surgery syndrome, with a 41-month follow-up: a retrospective case report. J Chiropr Med. 2018;17(4):256-63.

48. Oakley PA, Berry RH, Harrison DE. A structural approach to post-surgical laminectomy: a case study. J Vert Sublux Res. 2007;19:1-7.

49. Layton PD. Chiropractic care for a patient with subluxation \& unsuccessful surgery of the lumbar spine. J Vert Sublux Res. 2009;10:1-5.

50. Lisi AJ, Bhardwaj MK. Chiropractic high-velocity low-amplitude spinal manipulation in the treatment of a case of postsurgical chronic cauda equina syndrome. J Manipulative Physiol Ther. Nov;27(9):574-8.

51. Coulis CM, Lisi AJ. Chiropractic management of postoperative spine pain: a report of 3 cases. J Chiropr Med. 2013;12(3):168-75.

52. Welk AB, Werdehausen DN, Kettner NW. Conservative management of recurrent lumbar disk herniation with epidural fibrosis: a case report. J Chiropr Med. 2012;11(4):249-53.

53. Kruse RA, Cambron JA. Cox decompression chiropractic manipulation of a patient with postsurgical lumbar fusion: a case report. J Chiropr Med. 2011;10(4):255-60.

54. Demetrious J. Clinical imaging pearls. Posterior lumbar interbody fusion failure: a brief case presentation. J Acad Chiropr Orthoped. 2007;4(4):3-7.

55. Cox JM. Failed Back Surgical Syndrome - L1-2 and L5-S1 Disc Herniations Following L4- S1 Spinal Fusion: A Case Report. J Acad Chiropr Orthoped. 2009;6(3):17.

56. Gluck NI. Passive care and active rehabilitation in a patient with failed back surgery syndrome. J Manipulative Physiol Ther. 1996;19(1):41-7.

57. Hoiriis KT. Case report: management of post-surgical chronic back pain with upper cervical adjustment. Chiropr Res J. 1989;1(3):37-42.

58. Greenwood DM. Improvement in chronic low back pain in an aviation crash survivor with adjacent segment disease following flexion distraction therapy: a case study. J Chiropr Med. 2012;11(4):300-5.

59. Lamb KL. Sacroiliac joint dysfunction with associated piriformis syndrome mimicking intervertebral disc syndrome resulting in failed low back surgery. Chiropr Tech. 1997;9(3):128-32.

60. Peterson S, Hodges C. Lumbar lateral shift in a patient with interspinous device implantation: a case report. J Man Manip Ther. 2016;24(4):215-22.

61. Cornelson SM, Johnnie ED, Kettner NW. Neural mobilization in a 54-year-old woman with postoperative spinal adhesive arachnoiditis. $\mathrm{J}$ Chiropr Med. 2018;17(4):283-8.

62. Paris DJ, Schielke AL. Resolution of post-surgical low back pain in a patient with chronic cauda equina syndrome: a case study. J Acad Chiropr Assoc. 2017;14(4):43-9.

63. Eisenberg E by Vulfsons E, Waldman S M. Persistent Back Pain after Multiple Operations: Case Presentation From Israel With Commentaries From Austria and The Netherlands. J Pain Palliat Care Pharmacother. 2011;25(1):64-7.

64. Shaw TW. Chiropractic rehabilitation of the retraumatized postsurgical lumbar spine with radiculopathy. J Am Chiropr Assoc. 1996;33(3):71-4.

65. Francio V, Towery C, Davani S, Brown T. Spinal manipulation and therapeutic exercises in treating post-surgical resurgent lumbar radiculopathy. Oxf Med Case Rep. 2017;10:omx062.

66. Taylor DN. Spinal synovial cysts and intersegmental instability: a chiropractic case. J Manipulative Physiol Ther. 2007;30(2):152-7.

67. Kennedy L, Gonzales E, Corbin L. The effect of Curanderismo on chronic non-malignant pain: a case report. EXPLORE. 2016;12(4):263-7.

68. Keller G. The effects of massage therapy after decompression and fusion surgery of the lumbar spine: a case study. Int $\mathrm{J}$ Ther Massage Bodyw. 2012;5(4):3-8.

69. Vaillancourt PJ, Collins KF. Case report: management of post-surgical low back syndrome with upper cervical adjustment. Chiropr Res J. 1993;2(3):1-15.

70. Walker BF. Failed back surgery syndrome. COMSIG Rev. 1992;1(1):3-6.

71. Shapiro CM. The failed back surgery syndrome: pitfalls surrounding evaluation and treatment. Phys Med Rehabil Clin N Am. 2014;25(2):319-40.

72. Kim BJ, Ahn J, Cho H, et al. Rehabilitation with osteopathic manipulative treatment after lumbar disc surgery: A randomised, controlled pilot study. Int $\mathrm{J}$ Osteo Med. 2015;18(3):181-8.

73. Kim BJ, Kim T, Ahn J, Cho H, Kim D, Yoon B. Manipulative rehabilitation applied soon after lumbar disc surgery improves late post-operative functional disability: A preliminary 2-year follow-up study. J Back Musculoskelet Rehabil. 2017;30(5):999-1004.

74. Kim BJ, Ahn J, Cho H, Kim D, Kim T, Yoon B. Early individualised manipulative rehabilitation following lumbar open laser microdiscectomy improves early post-operative functional disability: A randomized, controlled pilot study. J Back Musculoskelet Rehabil. 2016;29(1):23-9.

75. Paanalahti K, Holm LW, Nordin M, Asker M, Lyander J, Skillgate E. Adverse events after manual therapy among patients seeking care for neck and/or back pain: a randomized controlled trial. BMC Musculoskelet Disord. 2014;15:77.

76. Walker BF, Hebert JJ, Stomski NJ, Clarke BR, Bowden RS, Losco B, et al. Outcomes of usual chiropractic. The OUCH randomized controlled trial of adverse events. Spine. 2013;38(20):1723-9.

77. Yin P, Gao N, Wu J, Litscher G, Xu S. Adverse Events of Massage Therapy in Pain-Related Conditions: A Systematic Review. Evid-Based Complement Altern Med. 2014;2014:480956.

78. Boyd C, Crawford C, Paat CF, Price A, Xenakis L, Zhang W. The impact of massage therapy on function in pain populations-a systematic review and meta-analysis of randomized controlled trials: Part I, patients experiencing pain in the general population. Pain Med. 2016;17(7):1353-75.

Page $27 / 30$ 
79. Corcoran KL, Bastian LA, Gunderson CG, Steffens C, Brackett A, Lisi AJ. Association between chiropractic use and opioid receipt among patients with spinal pain: a systematic review and meta-analysis. Pain Med. 2020;21(2):e139-45.

80. Whedon JM, Toler AWJ, Kazal LA, Bezdjian S, Goehl JM, Greenstein J. Impact of Chiropractic Care on Use of Prescription Opioids in Patients with Spinal Pain. Pain Med. 2020;pii:pnaa014.[epub ahead of print].

81. Whedon JM, Toler AWJ, Goehl JM, Kazal LA. Association Between Utilization of Chiropractic Services for Treatment of Low-Back Pain and Use of Prescription Opioids. J Altern Complement Med. 2018;24(6):552-6.

82. Nissen T, Wynn R. The clinical case report: a review of its merits and limitations. BMC Res Notes. 2004;7:264.

83. Moher D, Liberati A, Tetzlaff J, Altman DG, PRISMA Group. Preferred reporting items for systematic reviews and meta-analyses: the PRISMA statement. PLoS Med. 2009;6(7):e1000097.

84. Rathmell JP. A 50-Year-Old Man With Chronic Low Back Pain. JAMA. 2008 May 7;299(17):2066.

85. McGregor AH, Henley A, Morris TP, Doré CJ. An Evaluation of a Postoperative Rehabilitation Program After Spinal Surgery and Its Impact on Outcome: Spine. 2012 Apr;37(7):E417-22.

86. Aspegren DD, Burt AL. A study of postspinal surgery cases in chiropractic offices. J Manipulative Physiol Ther. 1994;17(2):88-92.

87. Ogutluler Ozkara G, Ozgen M, Ozkara E, Armagan O, Arslantas A, Atasoy MA. Effectiveness of physical therapy and rehabilitation programs starting immediately after lumbar disc surgery. Turk Neurosurg. 2015;25(3):372-9.

88. Estadt GM. Chiropractic/rehabilitative management of post-surgical disc herniation: a retrospective case report. J Chiropr Med. 2004;3(3):108-15.

89. Owers C, Wessely M. Chronic low back pain in a 24-year-old rugby player: Case discussion. Clin Chiropr. 2012;15(3-4):203-12.

90. Johansson A-C, Linton SJ, Bergkvist L, Nilsson O, Cornefjord M. Clinic-based training in comparison to home-based training after first-time lumbar disc surgery: a randomised controlled trial. Eur Spine J. 2009;18(3):398-409.

91. Abbott AD, Tyni-Lenné R, Hedlund R. Early rehabilitation targeting cognition, behavior, and motor function after lumbar fusion: a randomized controlled trial. Spine. 2010;35(8):848-57.

92. Teixeira MJ, Yeng LT, Garcia OG, Fonoff ET, Paiva WS, Araujo JO. Failed back surgery pain syndrome: therapeutic approach descriptive study in 56 patients. Rev Assoc Med Bras. 2011;57(3):282-7.

93. Demetrious J. Clinical pearl. Functional lumbar stenosis due to posterior lumbar interbody fusion. J Acad Chiropr Orthoped. 2008;5(2):12-6.

94. Greenwood J, McGregor A, Jones F, et al. Rehabilitation following lumbar fusion surgery: A systematic review and meta-analysis. Spine (Phila Pa. 1976;41(1):E28-36.

95. Picelli A, Buzzi MG, Cisari C, Gandolfi M, Porru D, Bonadiman S, et al. Headache, low back pain, other nociceptive and mixed pain conditions in neurorehabilitation. Eur J Phys Rehabil Med. 2016;52(6):14.

96. Ingber S. Iliopsoas myofascial dysfunction: a treatable cause of "failed" low back syndrome. Arch Phys Med Rehabil. 1989;70(5):382-6.

97. Chou R, Loeser JD, Owens DK, Rosenquist RW, Atlas SJ, Baisden J, et al. Interventional therapies, surgery, and interdisciplinary rehabilitation for low back pain: An evidence-based clinical practice guideline from the American Pain Society. Spine. 2009;34(10):1066-77.

98. Kjellby-Wendt G, Styf J. Early active training after lumbar discectomy. A prospective randomized, and controlled study. Spine (Phila Pa 1976). 1998;23(21):2345-51.

99. Soegaard R, Christensen FB, Lauersen I, Bünger CE. Lumbar spinal fusion patients' demands to the primary health sector: evaluation of three rehabilitation protocols. A prospective randomized study. Eur Spine J. 2006;15(5):648-56.

100. Reife MD, Coulis CM. Peroneal neuropathy misdiagnosed as L5 radiculopathy: a case report. Chiropr Man Ther. 2013;21(1):12.

101. Philadelphia Panel. Philadelphia Panel evidence-based clinical practice guidelines on selected rehabilitation interventions for low back pain. Phys Ther. 2001;81(10):1641-74.

102. Snowdon M, Peiris CL. Physiotherapy Commenced Within the First Four Weeks Post-Spinal Surgery Is Safe and Effective: A Systematic Review and Meta-Analysis. Arch Phys Med Rehabil. 2016;97(2):292-301.

103. Rushton A, Heneghan NR, Calvert M, Heap A, White L, Goodwin PC. Physiotherapy post lumbar discectomy: Prospective feasibility and pilot randomised controlled trial. PLOS ONE. 2015;10(11):e0142013.

104. Rushton A, Eveleigh G, Petherick E-J, Heneghan N, Bennett R, James G, et al. Physiotherapy rehabilitation following lumbar spinal fusion: a systematic review and meta-analysis of randomised controlled trials. BMJ Open. 2012;2(4):e000829.

105. Rushton A, Wright C, Goodwin P, Calvert M, Freemantle N. Physiotherapy rehabilitation post first lumbar discectomy: A systematic review and metaanalysis of randomized controlled trials. Spine. 2011;36(14):E961-72.

106. Erdogmus CB, Resch K-L, Sabitzer R, Müller H, Nuhr M, Schöggl A, et al. Physiotherapy-based rehabilitation following disc herniation operation: Results of a randomized clinical trial. Spine. 2007;32(19):2041-9.

107. Huysmans E, Goudman L, Van Belleghem G, De Jaeger M, Moens M, Nijs J, et al. Re: Return to work following surgery for lumbarradiculopathy-is there a need for postoperative rehabilitation? Spine J. 2018;18(12):2376-7.

108. Oosterhuis T, Costa LO, Maher CG, de Vet HC, van Tulder MW, Ostelo RW. Rehabilitation after lumbar disc surgery. Cochrane Database Syst Rev. 2014;14(3):CD003007.

109. McGregor AH, Probyn K, Cro S, Doré CJ, Burton AK, Balagué F, et al. Rehabilitation following surgery for lumbar spinal stenosis. A Cochrane review. Cochrane Database Syst Rev. 2014;39(13):1044-54. 
110. Rosomoff HL, Rosomoff RS. Comprehensive multidisciplinary pain center approach to the treatment of low back pain. Neurosurg Clin N Am. 1991;2(4):877-90.

111. Neblett R, Mayer TG, Brede E, Gatchel RJ. The effect of prior lumbar surgeries on the flexion relaxation phenomenon and its responsiveness to rehabilitative treatment. Spine J. 2014;14(6):892-902.

112. Ebenbichler GR, Inschlag S, Pflüger V, Stemberger R, Wiesinger G, Novak K, et al. Twelve-year follow-up of a randomized controlled trial of comprehensive physiotherapy following disc herniation operation. Clin Rehabil. 2015;29(6):548-60.

113. Monticone M, Giovanazzi E. Usefulness of a cognitive behavioural and rehabilitative approach to enhance long lasting benefit after lumbar spinal stenosis and degenerative spondylolisthesis surgery. A case report. Eur J Phys Rehabil Med. 2008;44(4):467-71.

114. Daley J. A 45-Year-Old Man With Low Back Pain and a Numb Left Foot, 1 Year Later. JAMA. 1999;281(16):1540.

115. Dellamonte NA. Alteration of spinal biomechanics after laminectomy with fusion. Chiropr Tech. 1997;9(2):62-6.

116. Gerwin R. Differential diagnosis of trigger points. J Musculoskelet Pain. 2004;12(3-4):23-8.

117. Johnson HH, Lamb KL. Thoracic disk herniation: A case report. J Manipulative Physiol Ther. 2001;24(5):367-8.

118. Jorgenson D, Confait H. Post-surgical back pain. BJC Case Studies. 1997;1(1):6-7.

119. McMorland G, Suter E, Casha S, du Plessis SJ, Hurlbert RJ. Manipulation or Microdiskectomy for Sciatica? A Prospective Randomized Clinical Study. J Manipulative Physiol Ther. 2010;33(8):576-84.

120. Marin GA. Lumbar disk protrusion. Evaluation and study of 600 diskectomeies with one to ten years followup. Internation surgery. 1974;59(3):154-5.

121. Miake-Lye IM, Mak S, Lee J, Luger T, Taylor SL, Shanman R, et al. Massage for Pain: An Evidence Map. J Altern Complement Med. 2019;25(5):475-502.

122. Puentedura EJ, Brooksby CL, Wallmann HW, Landers MR. Rehabilitation Following Lumbosacral Percutaneous Nucleoplasty: A Case Report. J Orthop Sports Phys Ther. 2010;40(4):214-24.

123. Ammendolia C, Côté $P$, Rampersaud YR, Southerst D, Budgell B, Bombardier $C$, et al. The boot camp program for lumbar spinal stenosis: a protocol for a randomized controlled trial. Chiropr Man Ther. 2016;24(1):25.

124. Fujii K, Abe T, Kubota S, Marushima A, Kawamoto H, Ueno T, et al. The voluntary driven exoskeleton Hybrid Assistive Limb (HAL) for postoperative training of thoracic ossification of the posterior longitudinal ligament: a case report. J Spinal Cord Med. 2017;40(3):361-7.

125. Ostelo RWJG, Köke AJA, Beurskens AJHM, de Vet HCW, Kerckhoffs MR, Vlaeyen JWS, et al. Behavioral-graded activity compared with usual care after first-time disk surgery: Considerations of the design of a randomized clinical trial. J Manipulative Physiol Ther. 2000;23(5):312-9.

126. Lewit K, Olsanska S. Clinical importance of active scars: Abnormal scars as a cause of myofascial pain. J Manipulative Physiol Ther. 2004;27(6):399402.

127. Ammendolia C, Chow N. Clinical outcomes for neurogenic claudication using a multimodal program for lumbar spinal stenosis: A retrospective study. J Manipulative Physiol Ther. 2015;38(3):188-94.

128. Herman PM, Hurwitz EL, Shekelle PG, Whitley MD, Coulter ID. Clinical scenarios for which spinal mobilization and nanipulation are considered by an expert panel to be inappropriate (and appropriate) for patients with chronic low back pain. Med Care. 2019;57(5):391-8.

129. Snider K, Snider E, DeGooyer B, Bukowski A, Fleming R, Johnson J. Retrospective medical record review of an osteopathic manipulative medicine hospital consultation service. J Am Osteopath Assoc. 2013;113(10):754-67.

130. Cupler ZA, Anderson MT, Stancik TJ. Thoracic spondylodiscitis epidural abscess in an afebrile Navy veteran: A case report. J Chiropr Med. 2017;16(3):246-51.

131. Kumar K, Malik S, Demeria D. Treatment of Chronic Pain with Spinal Cord Stimulation versus Alternative Therapies: Cost-effectiveness Analysis. Neurosurgery. 2002;51(1):106-16.

132. Colloca CJ, Polkinghorn BS. Chiropractic management of ehlers-danlos syndrome: a report of two cases. J Manipulative Physiol Ther. 2003;26(7):44859.

133. Lewis DD, Summers GK. Osteopathic manipulative treatment for the management of adjacent segment pathology. J Am Osteopath Assoc. 2017;117(12):782.

134. Cuka C, McDevitt AW, Porter-Hoke A, Karas S. Spinal manipulation after multiple fusions in an adult with scoliosis: a case report. J Man Manip Ther. 2019;27(2):115-24.

135. J Am Chiropr Assoc. Conservative management or surgery: The team approach. J Am Chiropr Assoc. 2003;40(2):8-14.

136. Forcum TL. Post disc herniation surgery rehab: A case study using Trigenics Applied Functional Neurology. Am Chiropr 2011:45-46.

137. Griffee SR, Prideaux C. Poster board 414: Acute pain management in rehabilitation unit: An exploratory study on the use of integrative medicine. Am J Phys Med Rehabil 2014;93(3 Suppl).

138. Imamura S. Efficacy of myofascial therapy in failed back surgery. Acupunct Electrother Res. 2006;31:157-92.

139. Stoll ST, Simmons SL. Inpatient rehabilitation and manual medicine. Phys Med Rehab. 2000;14(1):85-106.

140. Maffei P. Intérêt du massage dans le traitement de douleurs postopératoires précoces: étude contrôlée randomisée. Kinésithérapie Rev. 2014;14(145):1625.

141. Lapham-Yaun R, Castro K. Improved spinal alignment, chronic low back pain and improved quality of life in a 62-year-old patient undergoing chiropractic care following failed surgical syndrome: a case study \& review of the literature. Ann Vert Subluxation Res 2019:10-21.

\section{Figures}

Page 29/30 


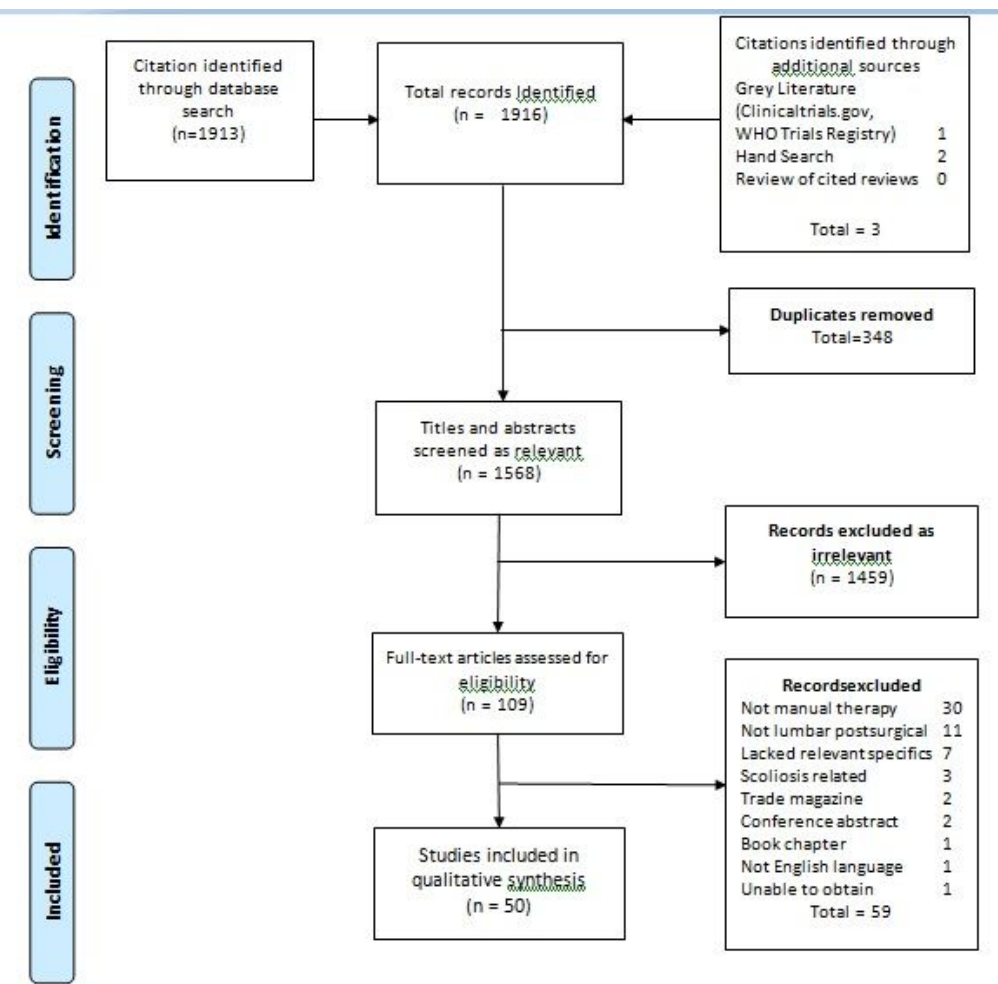

Figure 1

PRISMA Flow Diagram[83] 